\title{
AN EXPERIMENTAL INVESTIGATION OF SILICONE-TO-METAL BOND STRENGTH IN COMPOSITE SPACE DOCKING SYSTEM SEALS
}

\author{
by \\ MASON CHRISTIAN CONRAD
}

Submitted in partial fulfillment of the requirements

For the degree of Master of Science

Thesis Advisor: Dr. Joseph Prahl

Department of Mechanical and Aerospace Engineering

CASE WESTERN RESERVE UNIVERSITY

August 2009 


\section{CASE WESTERN RESERVE UNIVERSITY \\ SCHOOL OF GRADUATE STUDIES}

We hereby approve the thesis/dissertation of

Mason Conrad

candidate for the Master of Science degree *.

(signed) $\frac{\text { Dr. Joseph Prahl }}{\text { (chair of the committee) }}$
Dr. Iwan Alexander

Dr. Roger Quinn

(date) June 3, 2009

*We also certify that written approval has been obtained for any proprietary material contained therein. 


\section{Table of Contents}

Table of Contents ....................... ii

List of Tables . . . . . . . . . . . . . . . . . iv

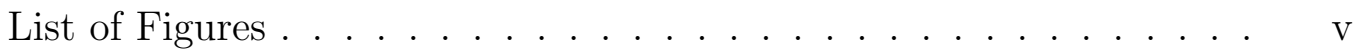

Acknowledgements ........................ vii

Abstract . . . . . . . . . . . . . . . viii

1 Introduction 1

1.1 The Orion Crew Exploration Vehicle . . . . . . . . . . . . . 1

1.2 The Low-Impact Docking System . . . . . . . . . . . . . . . 2

1.3 LIDS Main Interface Seal Requirements . . . . . . . . . . . . . . 4

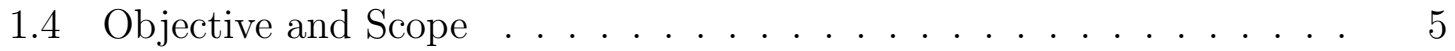

References ........................ 6

2 Background of the LIDS Main Interface Seal $\quad 7$

2.1 Historical Background of Docking Seal Bond Strength . . . . . . . . 7

2.2 Previous LIDS Main Interface Seal Work . . . . . . . . . . . . . . 10

2.3 Silicone Bond Strength Background . . . . . . . . . . . . . . . 12

References . . . . . . . . . . . . . . . . . . . . 14

3 Experimental Method 17

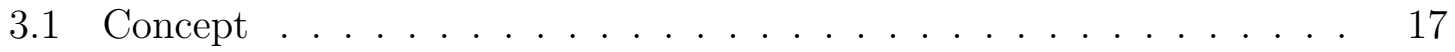

3.2 Adhesive Selection . . . . . . . . . . . . . . . . . . . 18

3.3 Test Fixture Design . . . . . . . . . . . . . . . . . . . . . . . . . . . . 19

3.4 Seal Test Specimens . . . . . . . . . . . . . . . . . . . . . . 21

3.5 Test $\operatorname{Rig} \ldots \ldots \ldots \ldots \ldots$

3.6 Experimental Procedure $\ldots \ldots \ldots \ldots \ldots$

3.6.1 RTV Bonding Sub-Procedure . . . . . . . . . . . . . . 26

3.6.2 Pull-Test Sub-Procedure . . . . . . . . . . . . . . . . 27

3.6.3 Post-Test Sub-Procedure . . . . . . . . . . . . . . . . . . . . . . 29

References . . . . . . . . . . . . . . . . . . . . . . . . . . . . 29 
4 Results and Discussion $\quad 31$

4.1 Background ......................... . . . . 31

4.2 Cohesive vs. Adhesive Failure . . . . . . . . . . . . . . . . . . . . . . . . . . . . . . . . 33

4.3 Uniform Adhesion Case . . . . . . . . . . . . . . . . . . . . . . . . . . . . . . . . . 38

4.3.1 Cohesive Failure Mode . . . . . . . . . . . . . . . . . 38

4.3.2 Adhesive Failure Mode . . . . . . . . . . . . . . . . . . . 44

4.3 .3 Mixed Failure Mode . . . . . . . . . . . . . . . . . . . 47

4.3.4 Comparison to Seal Adhesion Data . . . . . . . . . . . . . 50

4.4 Localized Adhesion Case . . . . . . . . . . . . . . . . . . . . . . . . . . . 52

4.4.1 Adhesive Failure Mode . . . . . . . . . . . . . . . . . . . 53

4.4 .2 Material Failure Mode . . . . . . . . . . . . . . . . 56

References . . . . . . . . . . . . . . . . . . 61

5 Conclusions $\quad 62$

5.1 Summary of Results . . . . . . . . . . . . . . . . . . . 62

5.2 Future Work . . . . . . . . . . . . . . . . . 64

A Measurement Uncertainty 65

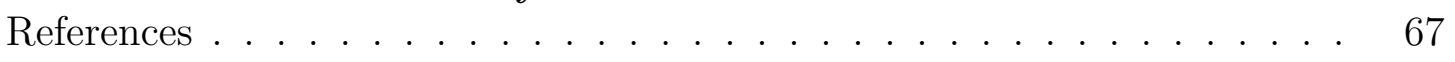

$\begin{array}{lr}\text { Bibliography } & 68\end{array}$ 


\section{List of Tables}

3.1 Seal specimen flaw levels and lengths . . . . . . . . . . . . . . 22

4.1 Summary of exact bond ratings as represented in Figures 4.2 and $4.3 \quad 37$ 


\section{List of Figures}

1.1 An artist's rendition of the Orion vehicle docking with the ISS (left) and LSAM (right) . . . . . . . . . . . . . . . 2

1.2 CAD images of the LIDS and main interface seal . . . . . . . . 4

2.1 Section views of historical dovetail-style groove interface seal designs . 8

2.2 Section view of CBM Gask-O-Seal . . . . . . . . . . . . . . . 9

2.3 Section view of LIDS main interface seal joint . . . . . . . . . . 11

2.4 Section views of nominal separation vs. seal bond failure . . . . . . 12

2.5 Photograph of localized disbond at an arbitrary location . . . . . . 13

2.6 Photograph of disbond occuring at bolt hole locations due to molding process . . . . . . . . . . . . . . . . . . 13

3.1 Photographs of adhesive selection testing . . . . . . . . . . . . 19

3.2 Section view of the test fixture concept . . . . . . . . . . . 19

3.3 CAD image of test fixture with inset view of RTV interface . . . . . 20

3.4 Exploded view of the setup for an inner bulb pull-test . . . . . . . . 21

3.5 Schematic of the definition of flaw length . . . . . . . . . . 22

3.6 Photographs of the load frame and components used for composite seal bond strength testing . . . . . . . . . . . . . . . . 24

3.7 Photograph of reflective tape for laser extensometer displacement measurement . . . . . . . . . . . . . . . . . . 25

3.8 Photograph of RTV applied to seal bulb for a localized case pull-test 27

3.9 Photographs of the bond strength pull-test procedure . . . . . . . 28

3.10 Graph of measured displacement vs expected LIDS interface separation profile . . . . . . . . . . . . . . . . . . . . . . . 29

4.1 Schematic showing the location of the 5 failure modes observed . . . 34

4.2 Bar chart summarizing the bond ratings of all uniform adhesion case test specimens . . . . . . . . . . . . . . . . . . 35

4.3 Bar chart summarizing the bond ratings of all localized adhesion case test specimens . . . . . . . . . . . . . . . . . 36 
4.4 Photograph of a fracture surface showing significant cohesive failure (CS), along with adhesive failure (AS) . . . . . . . . . .

4.5 Optical micrograph of fracture surface under 7.1X magnification showing cohesive failure (CS) patterns . . . . . . . . . . . . 40

4.6 Optical micrograph of fracture surface under 63X magnification showing small spots of cohesive failure (CS) invisible to the naked eye . . .

4.7 Graph of measured results for uniform load case exhibiting cohesive failure . . . . . . . . . . . . . . . . . 41

4.8 Bar chart representing failure load per circumferential inch of a single seal bulb . . . . . . . . . . . . . . . . . . . 43

4.9 Video frame of elastomer elongation just prior to failure . . . . . . . 44

4.10 Photograph of a representative area showing exclusively adhesive fail-

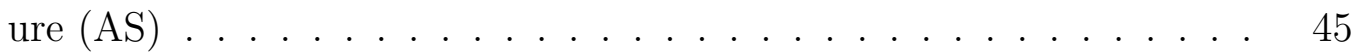

4.11 Optical micrograph of adhesive failure surface at $12.5 \mathrm{X}$ magnification 45

4.12 Graph of measured results for uniform load case exhibiting overwhelmingly adhesive failure . . . . . . . . . . . . 46

4.13 Video frame of the partial RTV failure in a uniform adhesion case leading to an altered load-displacement curve . . . . . . . . . 47

4.14 Photograph of the seal showing a combination of failure mode . . . 48

4.15 Photographs of fracture surfaces in a test showing mixed failure modes 49

4.16 Graph of measured results for uniform load case exhibiting mixed failure modes . . . . . . . . . . . . . . . . . . 50

4.17 Bar chart comparison of peak measured loads on adhesion tests and on bond strength tests . . . . . . . . . . . . . . . 52

4.18 Photograph of the complete removal of the seal bulb in one piece due to localized adhesion . . . . . . . . . . . . . . 54

4.19 Graph of measured results for localized adhesion case exhibiting adhesive failure . . . . . . . . . . . . . . . .

4.20 Video frame of the white peel line just visible at the base of the seal bulb when it separates from the groove bottom . . . . . . . 56

4.21 Optical micrograph of S0383-70 material failure (M) under 20X magnification near a geometric stress concentration . . . . . . . . 58

4.22 Optical micrograph of material fracture (M) surface under 16X magnification showing tear lines along seal bulb . . . . . . . . .

4.23 Video frame sequence showing the progressive material failure along the seal crown . . . . . . . . . . . . . . . .

4.24 Graph of measured results for localized adhesion case exhibiting S038370 material failure . . . . . . . . . . . . . . . .

4.25 Photograph of failure surface of $1 \mathrm{in}$. disbonded seal specimen with unexpected failure propagation $\ldots \ldots \ldots \ldots \ldots$ 


\section{ACKNOWLEDGEMENTS}

Many people were instrumental in the completion of this work. I thank my thesis advisor Dr. Joseph Prahl for his guidance and advice throughout my undergraduate and graduate years at Case. Also, I would like to thank Dr. Roger Quinn and Dr. Iwan Alexander for their valuable input as members of my thesis committee.

I am very grateful to Dr. Christopher Daniels of the University of Akron for his mentorship during my tenure as an intern and graduate student at NASA Glenn Research Center. I am also very appreciative of the rest of the LIDS team for their numerous contributions in the lab. I would like to extend special thanks to Mr. Dick Tashjian, who helped immensely in development and problem-solving during testing.

I thank Dr. Bruce Steinetz and Mr. Patrick Dunlap, Jr. for financial support through the Graduate Student Researchers Program at GRC, and for their guidance in this research. 


\title{
AN EXPERIMENTAL INVESTIGATION OF SILICONE-TO-METAL BOND STRENGTH IN COMPOSITE SPACE DOCKING SYSTEM SEALS
}

\author{
Abstract
}

by

\section{MASON CHRISTIAN CONRAD}

The National Aeronautics and Space Administration (NASA) is currently developing a new universal docking mechanism for future space exploration missions called the Low Impact Docking System (LIDS). In order to successfully mate two pressurized vehicles or structures in space, advanced seals are required at the interface to prevent the loss of breathable air to vacuum. The leading candidate LIDS main interface seal design is a composite assembly of silicone elastomer seal bulbs permanently molded into grooves in an electroless nickel-plated aluminum retainer. A composite seal of the style being developed has not been proven in a docking role in the history of manned spaceflight. High adhesive loads on the seal bulbs during undocking could potentially overcome the strength of the molded bond between the elastomer and the metal retainer. Bond failure would jeopardize the operation of the seal and docking port, and orbital debris could be liberated. The strength of the silicone-to-metal bond is a critical consideration for the new system, especially due to the presence of small areas of disbond created by the molding process. In the work presented herein, siliconeto-metal bonds of subscale seal specimens with different sizes of intentional disbond are destructively tensile tested. Tension is applied either uniformly on the entire seal circumference or locally in a single circumferential location. Bond failure due to uniform tension produces a wide scatter of observable failure modes and measured 
load-displacement behaviors. Although the ideal failure mode for the silicone-tometal bond is $100 \%$ cohesive failure of the material, the highest observed cohesion amount is $20 \%$ of bond area for the uniform loading condition. Localized tension produces failure either as immediate tearing of the elastomer material outside the bond region or as complete peel-out of the seal bulb in one piece. In intentionally flawed specimens, neither load case considered shows a correlation between disbond size and bond strength. 


\section{Chapter 1}

\section{Introduction}

\subsection{The Orion Crew Exploration Vehicle}

The National Aeronautics and Space Administration (NASA) is currently developing the Orion Crew Exploration Vehicle (CEV) for future manned missions to low-Earth-orbit (LEO), the Moon, and Mars. As part of the Constellation program, the CEV will replace the Space Shuttle as NASA's only vehicle capable of manned spaceflight. Although similar in shape to the Apollo command module, the Orion vehicle is unique in many respects including size, technical complexity, and mission capability. ${ }^{1}$

The CEV will be used in LEO missions to transport a crew of between three and six to the International Space Station (ISS) where it will remain docked, acting as an emergency escape vehicle, until it is used for crew return to Earth up to six months later. The initial lunar sortie mission scenario calls for the CEV to carry a crew of up to four to lunar orbit and remain there while the crew descends to explore the surface in the Lunar Surface Access Module (LSAM). The subsequent lunar outpost missions will require the CEV to remain in lunar orbit for up to six months while the construction of a permanent human base is carried out on the surface below. In both lunar mission architectures, an expendable ascent stage of the LSAM will dock with the Orion vehicle in lunar orbit to allow crew transfer before return to Earth in the 
CEV. Eventually, the Orion vehicle could be used to carry astronauts to Mars orbit, where it would remain for up to eighteen months. The CEV would then act as the reentry vehicle upon return to Earth. ${ }^{1}$

Although the goal of the Orion vehicle design is to support the broad array of missions planned, this body of work primarily applies to LEO missions to the ISS.

A critical operation for the $\mathrm{CEV}$ in all of the mission scenarios mentioned above is pressurized docking and undocking with other structures in space. The CEV will dock with the ISS, LSAM, and other future structures to allow cargo and crew transfer on orbit. Although several docking systems currently exist, a universal next-generation system is needed to meet the demands of the new spacecraft. The docking requirements of the Orion vehicle are currently being addressed through the development of the Low Impact Docking System (LIDS) ${ }^{2}$
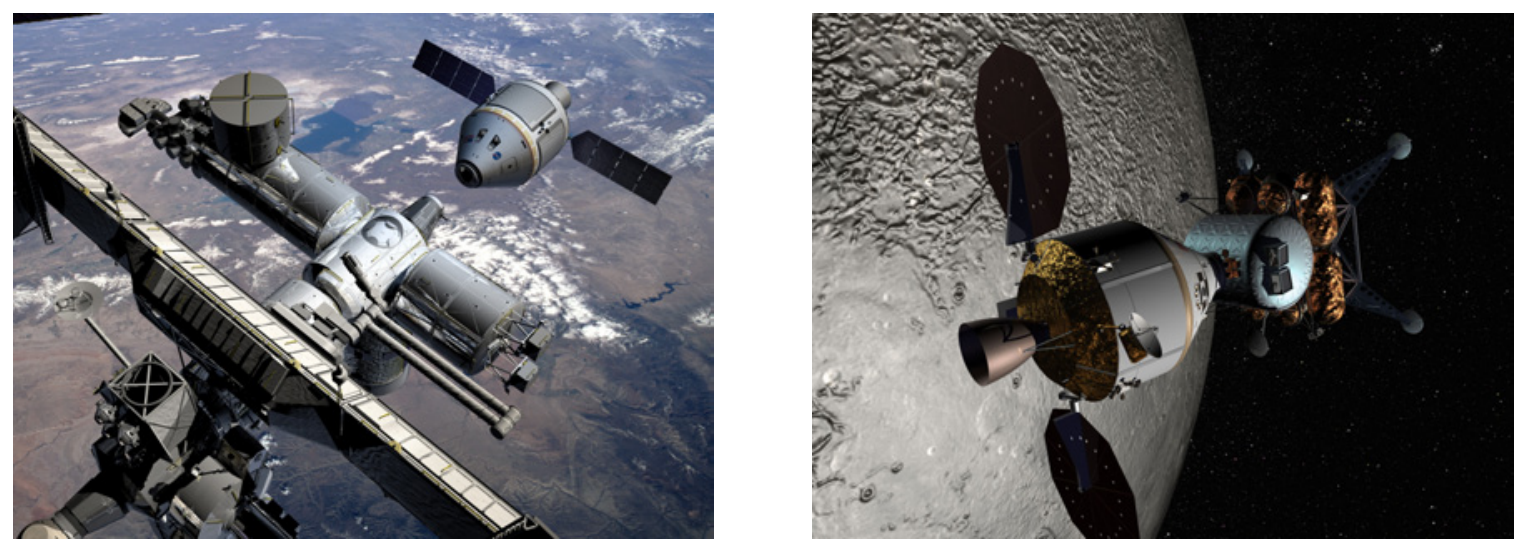

Figure 1.1: An artist's rendition of the Orion vehicle docking with the ISS (left) and LSAM (right)

\subsection{The Low-Impact Docking System}

The LIDS is being developed by NASA as the standard docking interface for all future space exploration missions. It is an improvement over existing systems because minimal impact loads are imparted on either vehicle during docking, whereas other systems require significant contact force to activate the capture mechanism. 
On the LIDS, an extendable load-sensing electromagnetic capture ring and closedloop feedback control are used to join two round docking interfaces at the periphery, allowing cargo and crew passage through the tunnel formed. During the mating of two vehicles, each equipped with a LIDS interface, one side assumes an active role and the other a passive role. The active LIDS extends its capture ring and attaches to the opposing passive unit. After a "soft" electromagnetic capture is achieved, the interface is aligned with a system of actuator arms, and then retracted. At this point, the pressure seals are compressed, structural attachments are made, and the docking process is completed. When the time comes for the vehicles to undock, the rigid latches are released and motorized spring-loaded mechanisms provide a separation force. Although the actual docking and undocking processes will be more complicated, this simplified explanation gives a general understanding of the sequence.

The system must function within size and weight limitations inherent in flight hardware. Currently, the LIDS must fit inside a cylindrical envelope 60 inches in diameter and 27 inches high and allow a crew passage 32 inches in diameter. The baseline active LIDS configuration on CEV is to weigh no more than approximately $550 \mathrm{lbs}$, although different configurations may vary significantly in weight. Aside from the advantages provided by the low-impact characteristics of the docking process, the LIDS must allow safe crew and cargo passage while docked, as well as other functions including data transfer and status indication. The size, weight, and functional requirements of the LIDS introduce the need for sophisticated components at all levels of the system. 


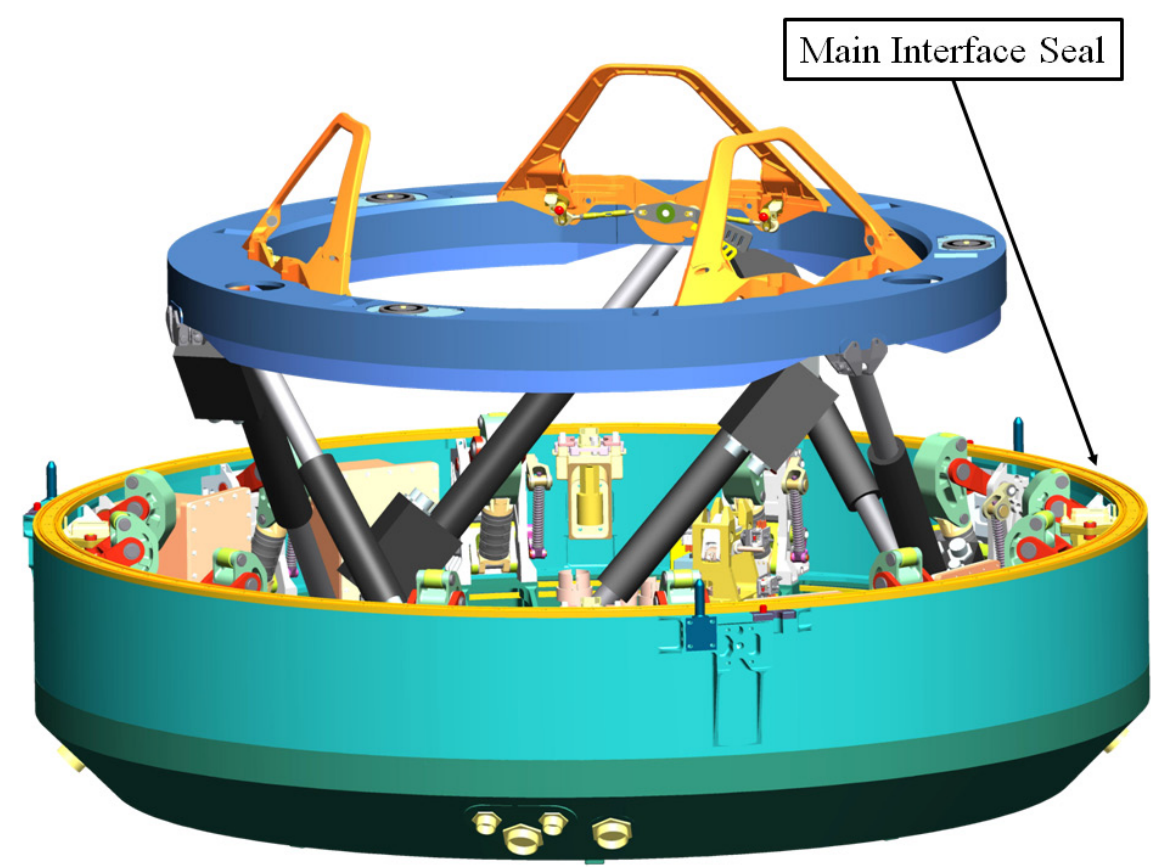

(a) Low Impact Docking System

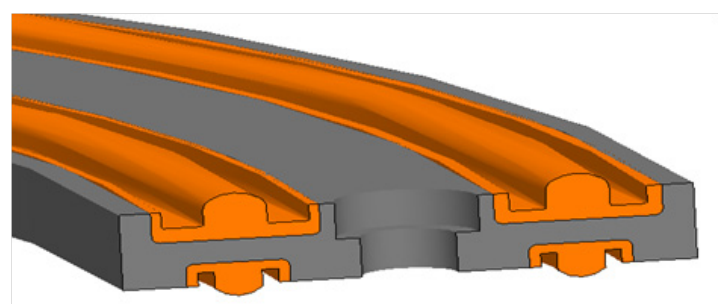

(b) Candidate design for composite seal

Figure 1.2: CAD images of the LIDS and main interface seal

\subsection{LIDS Main Interface Seal Requirements}

The successful operation of the LIDS requires an advanced seal at the mating interface to prevent the loss of breathable cabin air to vacuum. The performance requirements of the main interface seal present considerable design challenges that must be overcome. The LIDS main interface seal is being developed at NASA Glenn Research Center (GRC) in Cleveland, OH.

A key design challenge for the main interface seal is its operating environment 
which includes prolonged exposure to atomic oxygen (AO), Ultraviolet (UV) and ionizing radiation, vacuum pressure, and potential micro-meteoroid/orbital debris (MMOD) impact damage. The exposure temperature range expected is approximately $-100^{\circ} \mathrm{C}$ to $125^{\circ} \mathrm{C}$. Also, NASA requires that all spacecraft materials exposed to hard vacuum meet low-outgas requirements, as liberated material can condense on other external objects and affect their functionality.

In the docked configuration, the seal must exhibit extremely low leakage rates to decrease the amount of replacement air that must be stored onboard. The seal is also required to be redundant, so that a single failure does not defeat the sealed interface. The force required to fully compress the seal during docking must be minimized to avoid the weight penalty of large latches and actuators. Similarly during undocking, the force required to separate the interface must be minimized to allow smaller separation mechanisms to be used. The seal design involves tradeoffs between desirable leakage and load performance parameters while staying within system limits.

During undocking, it is critical that seal material is not dislodged by the action of separation as this could liberate orbital debris and damage the seal, potentially impairing sealing ability and redundancy. Seal material left attached to the interface could also disable the docking port. Therefore, such an event must be avoided, and is being addressed individually in the current work.

\subsection{Objective and Scope}

Although substantial effort has been put into the development of the LIDS main interface seal, the pull-out risk has not been adequately investigated. This thesis attempts to determine the ability of the current candidate seal design to avoid a seal bulb pull-out as described above. Future testing can use this work as a benchmark for whether adhesion under a given condition presents a likelihood of a seal bond failure. As the LIDS main interface seal is still under development, comparison of measured adhesive loads to actual destructive tests will be a useful tool in the acceptance of the final design. Experimental results are given for subscale tests in 
which the seal is intentionally pulled out of the groove under two different separation scenarios. The effects of manufacturing flaws on pull-out risk are considered by testing specimens with intentionally disbonded areas. This work is limited to the leading candidate design under ambient conditions. The chemical and molecular interactions of the bonding interface are not discussed due to the proprietary nature of the compounds under consideration. Instead the bond strength is analyzed using measured load-displacement data and visual obvervations of the fracture surfaces to gain an understanding of the strength of the seal bond.

\section{References}

[1] NASA's Exploration Systems Architecture Study Final Report, 2005.

[2] J. L. Lewis, M. B. Carroll, R. H. Morales, and D. L. Thang, Androgynous, reconfigurable closed loop feedback controlled Low Impact Docking System with load sensing electromagnet capture system, U.S. Patent no. 6354540, 2002. 


\section{Chapter 2}

\section{Background of the LIDS Main Interface Seal}

\subsection{Historical Background of Docking Seal Bond Strength}

It is valuable to briefly consider past approaches on the issue of interface seal retention during undocking for perspective on the LIDS application. There have been decades of docking experience in the U.S., Soviet, and Russian space programs from which to draw comparisons to the LIDS, but only a few informative examples are given here.

The Apollo docking system's interface seal consisted of hollow elastomer bulb seals installed into dovetail grooves on the docking ring of the command module, as seen in Figure 2.1a. ${ }^{1}$ A variation of this concept was used for the American docking system in the Apollo-Soyuz Test Project (ASTP), whereby a retainer ring clamped down on ridges on the bulb seals, as shown in Figure 2.1b; the Soviet system used a dovetail groove with a bulb seal installed. ${ }^{2}$ This approach provides for seal retention during undocking by physically housing the bulb seals with the shape of the groove and thus no bond is required between the elastomer and metal. The Russian-built Androgynous Peripheral Attach System (APAS) and probe-drogue docking systems have implemented forms of the dovetail-style groove design shown in Figure 2.1c for 
several decades with general success.

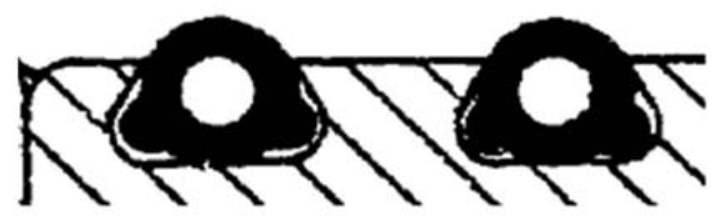

(a) Apollo : Hollow seals (black) in dovetail grooves $^{1}$

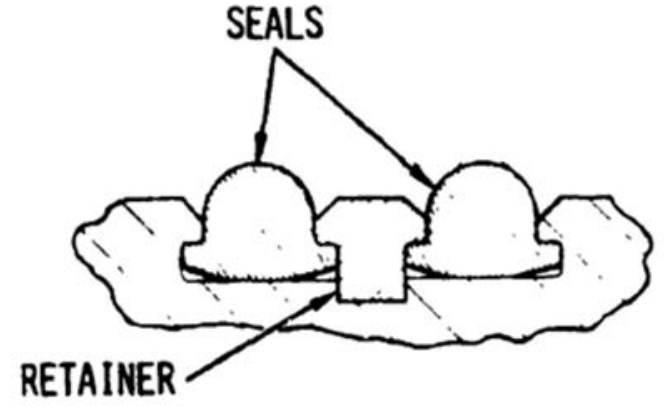

(b) ASTP : Retainer clamped onto seal ridges ${ }^{2}$

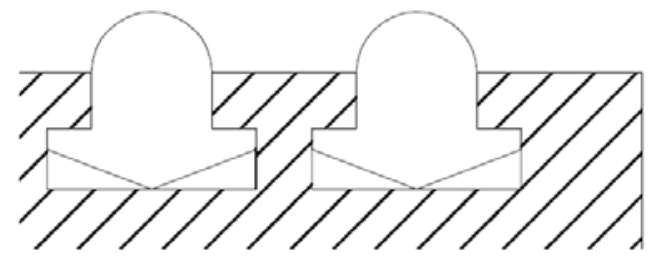

(c) APAS : Bulb seals in dovetail-style grooves

Figure 2.1: Section views of historical dovetail-style groove interface seal designs

There has been one known failure of the dovetail-style groove design. When it undocked in 2001, the Progress M45 cargo vehicle (No. 245) left a piece of the rubber interface seal on the surface of the Zvezda service module docking port on the ISS. This prevented the next Progress vehicle from achieving hard dock at that port and it was necessary for two ISS Expedition Three cosmonauts to perform an extravehicular activity (EVA) to remove the debris. On the ground, the launch of Shuttle mission STS-108 was delayed until the problem could be resolved. It was proposed that the cause of the seal failure was increased localized adhesion due to aggressive surface contaminants on the interface, possibly originating from exhaust plume impingement or fluid dumps. The length of time in the docked configuration (91 days) also may 
have contributed to the failure. This type of incident must be avoided in the LIDS main interface seal.

An alternate seal retention approach has demonstrated success as the static interface seal in the Common Berthing Mechanism $(\mathrm{CBM})^{3}$, the system used to mate pressurized ISS modules. Berthing refers to the joining of two bodies using a control arm for vehicle manipulation while docking is defined as the mating of two free-flying vehicles under their own control. The CBM uses a composite Gask-O-Seal ${ }^{T M}$ made by Parker-Hannifin Corporation, which relies on a molded bond between the elastomer and metal rather than physical entrapment. Figure 2.2 shows that the grooves on the CBM seal are perpendicular to the interface and therefore there is no physical feature to hold the elastomer seals in the groove. The primary benefit of the Gask-O-Seal ${ }^{T M}$ is that it can exhibit good performance characteristics while being easy to install and replace. Despite the reliability shown thus far by this design, there are no published studies that systematically investigate the elastomer bond strength in the CBM seal.

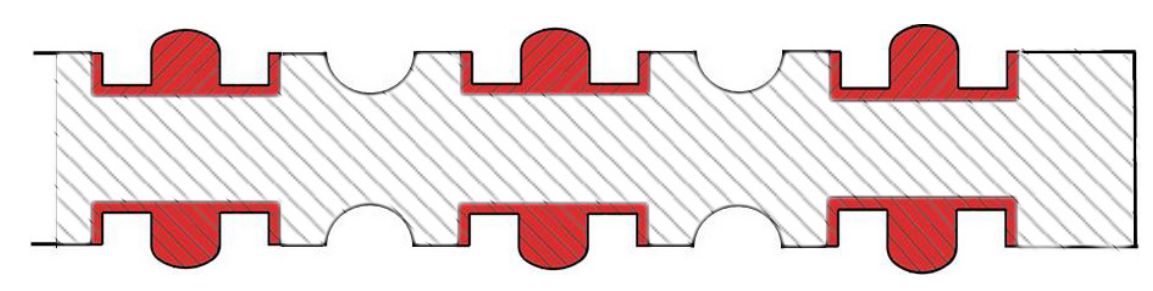

Figure 2.2: Section view of CBM Gask-O-Seal

Past approaches to the question of seal retention during undocking provide context for the present work. The Progress undocking incident shows that an interface seal can in fact pull out of a dovetail-style groove under certain conditions. Composite seal designs have been proven in a static berthing role, but have yet to be expanded into the operation of docking. 


\subsection{Previous LIDS Main Interface Seal Work}

The initial design phase of the LIDS main interface seal consisted of material evaluations investigating several elastomeric candidates' capability of performance in the space environment by Daniels et al. ${ }^{4,5}$ Metallic candidates were also considered by Oswald et al. ${ }^{6}$ Although the metallic materials tested could meet the leakage and compression requirements, metallic seals would be difficult to manufacture on the scale required for the interface seal (approximately 58 inch diameter). A study by deGroh et al found that the elastomer candidate materials demonstrated acceptable performance characteristics after exposure to AO exposure and UV radiation. ${ }^{7}$ The silicone elastomer S0383-70, made by Parker Hannifin Corporation, was chosen as a leading candidate material for the LIDS seal.

The leading candidate design is the Gask-O-Seal ${ }^{T M}$, based on its successful use on the CBM. The Gask-O-Seal ${ }^{T M}$ design consists of an aluminum annular retainer with four silicone elastomer bulbs permanently vacuum-molded into grooves on either side. A section view of the sealing interface is shown in an uncompressed state for clarity in Figure 2.3. The double-bulb layout on each side provides redundancy and the ability to check for leaks prior to launch. The smaller back-side bulbs statically mate with a flange on the vehicle carrying the active LIDS while the larger front-side bulbs are compressed during docking. 


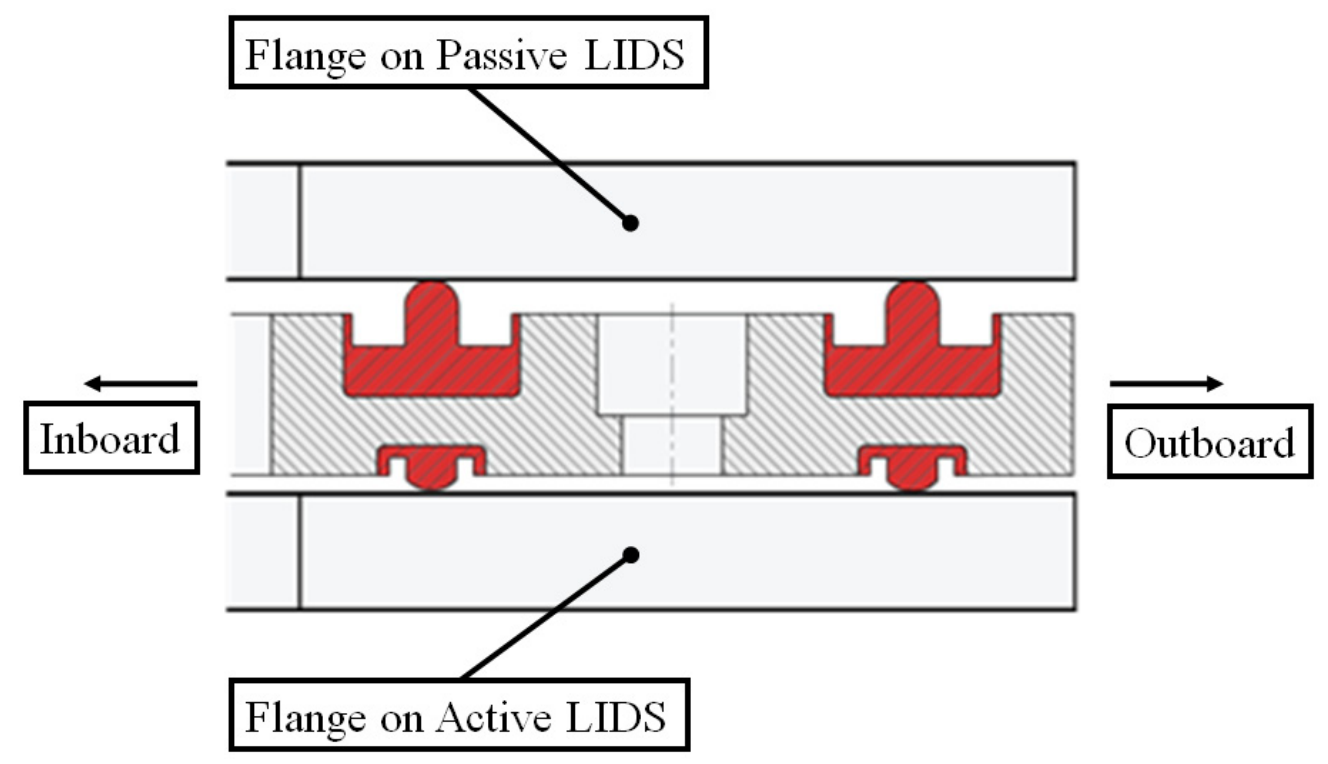

Figure 2.3: Section view of LIDS main interface seal joint

Investigations of subscale Gask-O-Seals ${ }^{T M}$ (approximately 12 inch diameter) have been conducted to guide the seal design. Subscale tests allow the numerous factors that affect seal performance to be investigated without the high cost and complexity required of full-scale seal tests. The performance characteristic that relates to the current work is the adhesion load generated during separation. Subscale seal adhesion has been shown to be affected by the silicone durometer ${ }^{8}$, level of AO pretreatment ${ }^{9}$, and cross sectional geometry ${ }^{10}$.

The results from subscale tests are used to estimate the adhesion values that a full-scale seal would produce and design decisions are made accordingly. A metric commonly used is the adhesion per length unit of seal, e.g. lbf/in. This value is multiplied by the circumference of a full-scale design to calculate the equivalent adhesion at full-scale. This can be taken as a reasonable approximation because the seal width is small compared to the seal diameter. Although the full-scale values are what ultimately must meet the mission requirements, the subscale tests are a critical element to the seal development. 


\subsection{Silicone Bond Strength Background}

As the adhesion of the S0383-70 material has been shown to be significant ${ }^{9}$, the possibility exists that the silicone-to-retainer bond might fail before the adhesion at the interface "releases", as shown in Figure 2.4. A bond failure could liberate silicone material as orbital debris, which could then land on and contaminate other external components of the vehicle. Seal fragments could be left behind on the mating flange, disabling the docking port as in the Progress incident. For ISS mission scenarios, a disabled docking port could affect launch schedules and would likely require an EVA to repair. In addition, the seal could lose sealing ability and redundancy due to the missing sections. A failure during a lunar mission could prove more severe as the inability of the surface lander to successfully re-dock with the CEV prior to Earth return would put the crew at substantial risk.

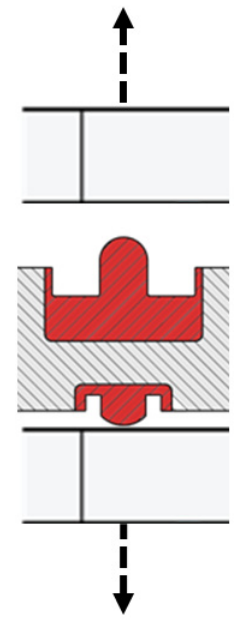

(a) Nominal separation

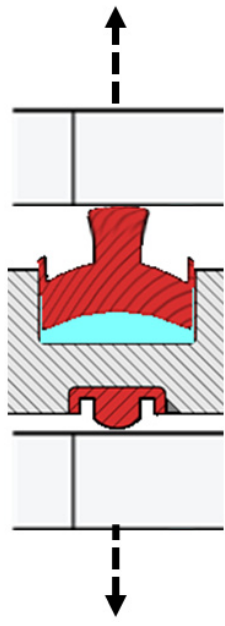

(b) Seal bulb pull-out

Figure 2.4: Section views of nominal separation vs. seal bond failure

This risk is compounded by the fact that the bond between the silicone and metal retainer is imperfect. After the metal retainer is fabricated, a priming agent is applied to the groove surfaces prior to silicone molding. Once the silicone cures in place in the grooves, it is permanently bonded to the retainer. Localized disbond occurs where 
the silicone separates from the metal, as seen in Figure 2.5. This is visible to some extent on all seal specimens received thus far, as the injection of the silicone during molding disrupts bonding at the bolt hole injection locations. An example of this type of disbond is shown in Figure 2.6. Therefore, it is reasonable to assume some amount of localized silicone disbond on the main interface seal on the CEV. Although disbonded regions may be small relative to seal circumference, it is unknown what effect they have on overall bond strength.

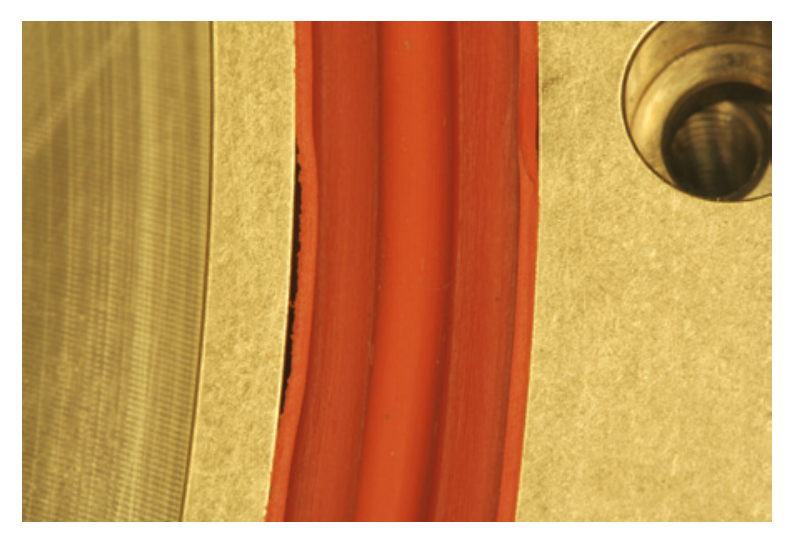

Figure 2.5: Photograph of localized disbond at an arbitrary location

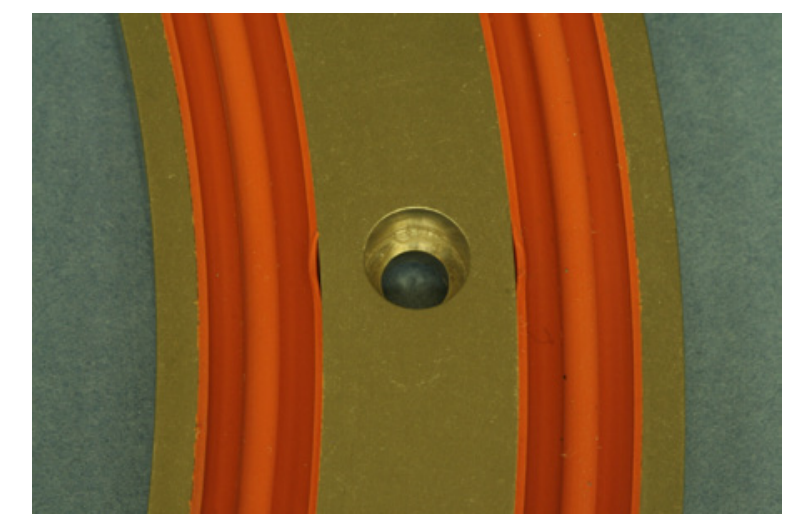

Figure 2.6: Photograph of disbond occuring at bolt hole locations due to molding process

Another aspect of the seal design that affects the silicone bond strength is the choice of material for the metal surface. As adhesion is strongly dependant on the 
surface chemistry involved, different metal substrate surfaces produce different bond strengths. The current baseline design is an aluminum retainer ring that has been electroless nickel (eNi) plated. The eNi-plating of the retainer is an option proposed to satisfy the electrical bonding requirements for the LIDS interface, which limit the amount of DC resistance in the joint to create a uniform, low-impedance vehicle skin in order to protect against RF interference.

Other factors also influence the silicone bond strength, but this work is limited to an investigation of the current baseline design only. The primary benefit of testing seal specimens is the ability to directly compare the bond strength results with data from adhesion tests of seals with the same geometry, elastomer compound, and separation rate. It would be more difficult to predict full-scale bond strength values using standardized test specimens because the load profile and geometry vary greatly from the seal's. Equally important is the desire to validate the manufacturing process used for actual seal samples, not laboratory test specimens. For example, a bond between the S0383-70 and retainer may be more or less difficult to produce in a given seal design's geometry than it would be in a standardized test specimen. It is most desirable to test the bond strength of seals produced with the same processes as eventual flight hardware.

The LIDS main interface seal development program at GRC includes plans for long-duration seal performance tests in which subscale seal specimens are compressed for 7 months in a vacuum chamber. The time frame is the expected longevity of a single CEV's tenure as an escape capsule docked at the ISS. The results of this future work will likely be the highest adhesion levels seen thus far and the ability for direct comparisons to this thesis will be very valuable to the development process.

\section{References}

[1] J. R. Finkbeiner, P. H. Dunlap, Jr, B. M. Steinetz, and C. C. Daniels. Review of seal designs on the Apollo spacecraft. Journal of Spacecraft and Rockets, 45(5), 2008. 
[2] W. L. Swan, Jr. Apollo-Soyuz Test Project docking system. In Proceedings of the 10th Aerospace Mechanisms Symposium, pages 26-37, 1976.

[3] Erik Illi. Space station freedom common berthing mechanism. In Proceedings of the 26th Aerospace Mechanisms Symposium, pages 281-296, 1992.

[4] C. C. Daniels, J. J. Oswald, P. H. Dunlap, Jr, and B. M. Steinetz. Elastomeric seal development of Advanced Docking/Berthing System. In 2005 NASA Seal/Secondary Air System Workshop, pages 465-484, 2005.

[5] C. C. Daniels, H. C. deGroh, III, P. H. Dunlap, Jr, J. R. Finkbeiner, B. M. Steinetz, M. B. Bastrzyk, J. J. Oswald, B. A. Banks, J. A. Dever, S. K. Miller, and D. L. Waters. Characteristics of elastomer seals exposed to space environments. In 43rd AIAA/ASME/SAE/ASEE Joint Propulsion Conference and Exhibit, number AIAA 2007-5741, 2007.

[6] J. J. Oswald, C. C. Daniels, P. H. Dunlap, Jr, and B. M. Steinetz. Metallic seal development of Advanced Docking/Berthing System. In 2005 NASA Seal/Secondary Air System Workshop, pages 507-523, 2005.

[7] H. C. deGroh, III, C. C. Daniels, P. H. Dunlap, Jr, S. Miller, J. Dever, D. Waters, J. J. Oswald, and B. M. Steinetz. Space environment's effects on seal materials. In 2006 NASA Seal/Secondary Air System Workshop, pages 507-523, 2006.

[8] C. C. Daniels, J. J. Oswald, M. B. Bastrzyk, I. Smith, P. H. Dunlap, Jr, and B. M. Steinetz. Experimental investigation of elastomer docking seal compression set, adhesion, and leakage. In AIAA SPACE 2007 Conference and Exposition, number AIAA 2007-6197, 2007.

[9] H. C. deGroh, III, S. K. R. Miller, I. Smith, C. C. Daniels, and B. M. Steinetz. Adhesion of silicone elastomer seals for NASA's Crew Exploration Vehicle. Technical Report NASA TM-2008-215433, National Aeronautics and Space Administration, 2008. 
[10] I. Smith and C. C. Daniels. Experimental investigation into medium scale LIDS seal performance. In 2007 NASA Seal/Secondary Air System Workshop, 2007. 


\section{Chapter 3}

\section{Experimental Method}

\subsection{Concept}

The development of a means to test the bond strength of fabricated composite seals was a considerable challenge. In order to test the silicone-to-metal bond, a stronger joint is required on the top surface of the seal bulb than the bond formed during vulcanization of the elastomer to the metal. A weaker link at the top of the bulb would cause failure to occur there and produce a measurement of the minimum of the seal bond strength, but no other information about the silicone-to-metal interface would be obtained. Mechanical attachment methods such as pinching the bulb were dismissed as that could introduce localized stress to the bulb that would not occur during a normal separation. It was decided to use an adhesive on the top of the seal bulb to attach it to the test fixture for pull-out. The bonding technique required significant development and practice so that it could be used with confidence on the one-time destructive tests of seal specimens.

Two separation scenarios are tested using the same adhesive and fixturing. In the uniform adhesion case, the adhesive is applied to the entire circumference of the seal bulb. This simulates maximum seal-to-flange adhesion on the entire sealing surface and results can be compared to the levels of adhesion found by Daniels et $\mathrm{al}^{1}$ and deGroh et $\mathrm{al}^{2}$ in the seal-on-plate test configuration. The analysis of the Progress 
incident indicated that increased adhesion is also possible in a localized area. The localized adhesion scenario is tested by only applying the adhesive to a 1 inch length of the circumference. Tests on intentionally flawed seals position the adhesive directly on the disbonded area, representing a true worst-case load profile on the seal-to-retainer bond.

\subsection{Adhesive Selection}

A series of tests was done in order to find a suitable adhesive for bonding a test fixture to the seal bulb. The tests consisted of adhering each side of a 0.5 inch diameter S0383-70 button specimen to matching test plates, then allowing the adhesive to cure. Once cured, the two small plates were pulled apart in a load frame and the peak load measured. Figure 3.1a shows a representative test with the silicone button and adhesive between two horizontal plates. Using ASTM recommendations as a guide $^{3}$, different surface preparation techniques were experimented with for anodized aluminum, copper, and carbon steel plates. Carbon steel was chosen for the eventual seal test fixture because adequate bonding with the adhesive could be achieved with relatively easy surface preparation. A summary of the surface preparation technique as part of the overall setup process is given in Section 3.6.1.

A cyanoacrylate ("Super Glue") and two room-temperature-vulcanizing (RTV) silicone adhesives were tested with the objective being to produce a bond stronger than the S0383-70 material. The adhesive that proved capable of this was Dow Corning 3145 RTV MIL-A-46146 (Gray), shown in Figure 3.1b. As seen in the figure, the button sample tears in the midsection, indicating the bond on the top and bottom with the RTV is stronger than the silicone material itself. Upon finding an acceptable adhesive and compatible metal, the seal test fixture could be designed. 


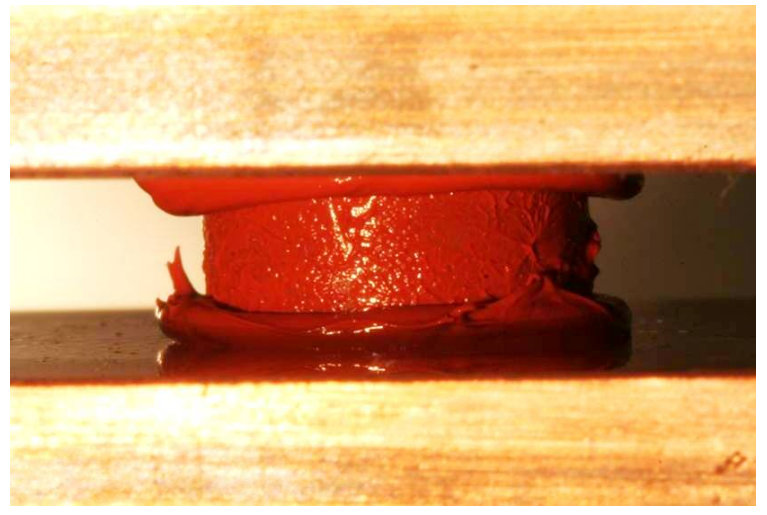

(a) Adhesive selection test setup prior to being pulled apart

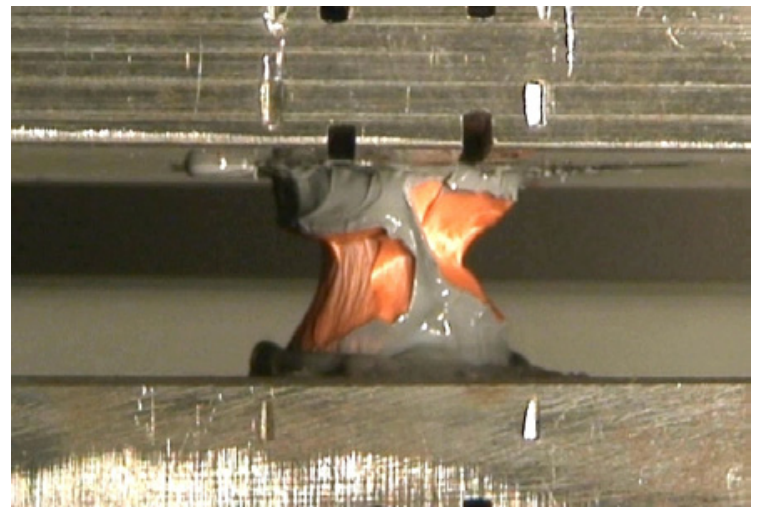

(b) Adhesive selection test exhibiting material failure in the silicone (red) due to superior bonds on the top and bottom of the button-shaped sample with the RTV (gray)

Figure 3.1: Photographs of adhesive selection testing

\subsection{Test Fixture Design}

In order to maximize the strength of the RTV-to-seal bond, the designed test fixture uses the geometry of the seal to full advantage. As seen in Figure 3.2, by matching the profile of the seal bulb, the area available for RTV bonding is maximized. This is especially beneficial for the vertical sides of the seal crown because the adhesive is loaded in shear where it is strongest.

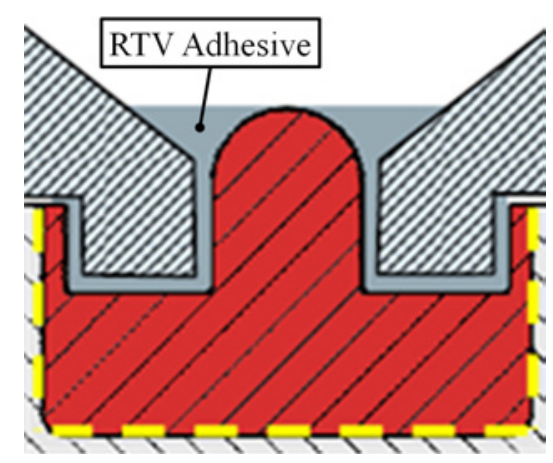

Figure 3.2: Section view of the test fixture concept to maximize adhesive bonding area by matching the seal bulb profile. The seal bond being tested is shown in dashed yellow lines. 
Two rings having the necessary cross-section are designed to perform one bulb test at a time, necessitating different ring sizes for the inner and outer seal bulbs. A threaded through-hole pattern on the top of the rings is used for attachment. Figure 3.3 shows a view of the joint created by the rings and the top surface of the seal bulb. The top surfaces of the rings and the seal are bolted to identical 1 inch thick stainless steel test plates, shown in yellow in Figure 3.3. Figure 3.4 shows an exploded view of the assembly needed to set up the RTV-to-seal bond for an inner bulb test. The setup and pull-test procedures are described in more detail in Section 3.6.

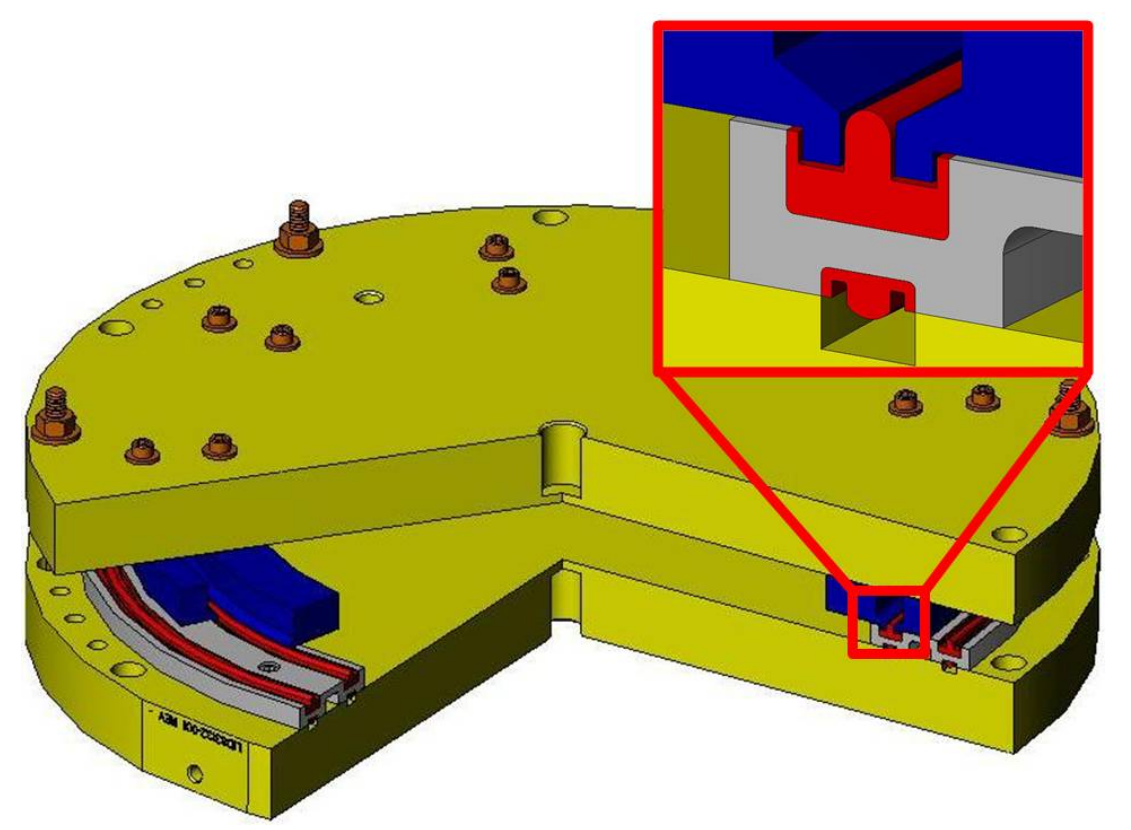

Figure 3.3: CAD design of the test fixture. The inset image shows the ring surface matching that of the seal bulb to maximize RTV bond area. 


\begin{tabular}{|l|l|}
\hline 1 & Upper 1" plate \\
\hline 2 & Fixture ring \#1 \\
\hline 3 & Fixture ring \#2 \\
\hline 4 & Seal test specimen \\
\hline 5 & Lower 1" plate \\
\hline 6 & $\# 8-32 \times 1.375 "(16)$ \\
\hline 7 & $\frac{1}{2}-20$ nuts $(6)$ \\
\hline 8 & $\# 8-32 \times 0.25 "(12)$ \\
\hline 9 & $\frac{1}{2}-20 \times 3.5 "(6)$ \\
\hline
\end{tabular}

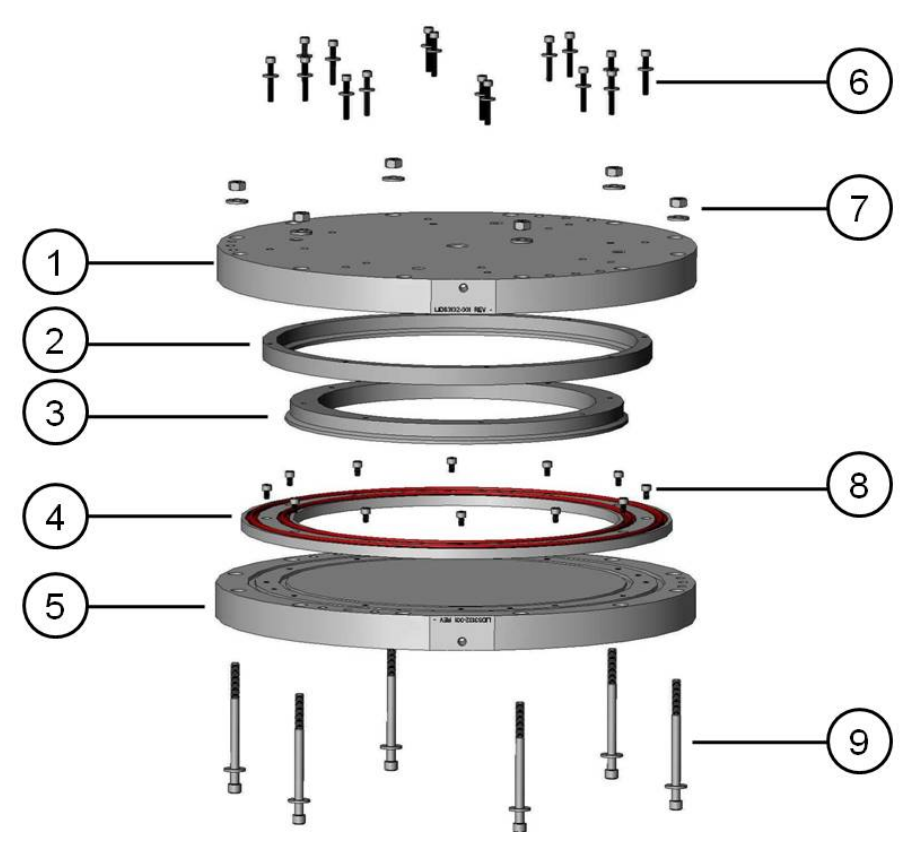

Figure 3.4: Exploded view of the setup for an inner bulb pull-test

\subsection{Seal Test Specimens}

The seal specimens in this study are fabricated from a slightly modified form of the manufacturer's normal processes in order to create varying levels of intentional "flaw" or disbond. By masking a specified length of the groove when applying the 
priming agent, disbond is encouraged on the unprimed area. Figure 3.5 shows the definition of flaw length in relation to the seal. All seals include eNi plating as the bonding surface for the elastomer.

Seal specimens fabricated with four levels of intentional flaw from 0 to 1 inch in length are investigated. The unflawed specimens are the product of the normal fabrication process with the entire groove primed. Table 3.1 gives the sizes of intentional disbond in the received test specimens.

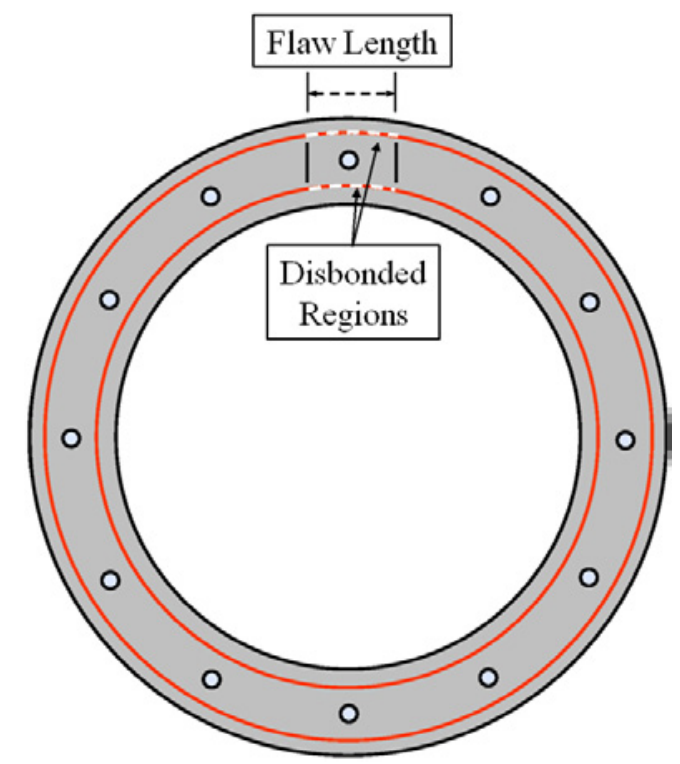

Figure 3.5: Schematic of the definition of flaw length

\begin{tabular}{|c||c|c|}
\hline & Flaw Level & Flaw Length (in.) \\
\hline \hline a. & Control & 0 \\
\hline b. & 1 & 0.25 \\
\hline c. & 2 & 0.5 \\
\hline d. & 3 & 1 \\
\hline
\end{tabular}

Table 3.1: Seal specimen flaw levels and lengths

It should be noted that the intentional disbonds in the test specimens represent a worst-case scenario. Typically the molding process produces some level of disbond 
visible at the edge of the groove on the side wall, as seen in Figure 2.5. Although disbond could occur at the bottom of the groove, it is very unlikely that a seal produced with the normal process would have both side walls and the entire groove bottom completely disbonded at the same circumferential location.

The seal specimens exhibit other disbonds in addition to the intentionally created flaws. The small disbonds at the bolt hole locations are evident as explained in Section 2.3 and other flaws of various length are visible as well. From visible inspection only, it is difficult to determine whether a given disbond extends past the top of the side wall to the groove bottom.

\subsection{Test Rig}

The pull-tests are performed in an Instron 5584 electromechanical load frame capable of 46.5 inches of crosshead travel. The frame is equipped with an alignment fixture for both angular and concentricity adjustments of the upper rod with respect to the lower rod. Figure 3.6 shows the test rig with all of the components involved. 


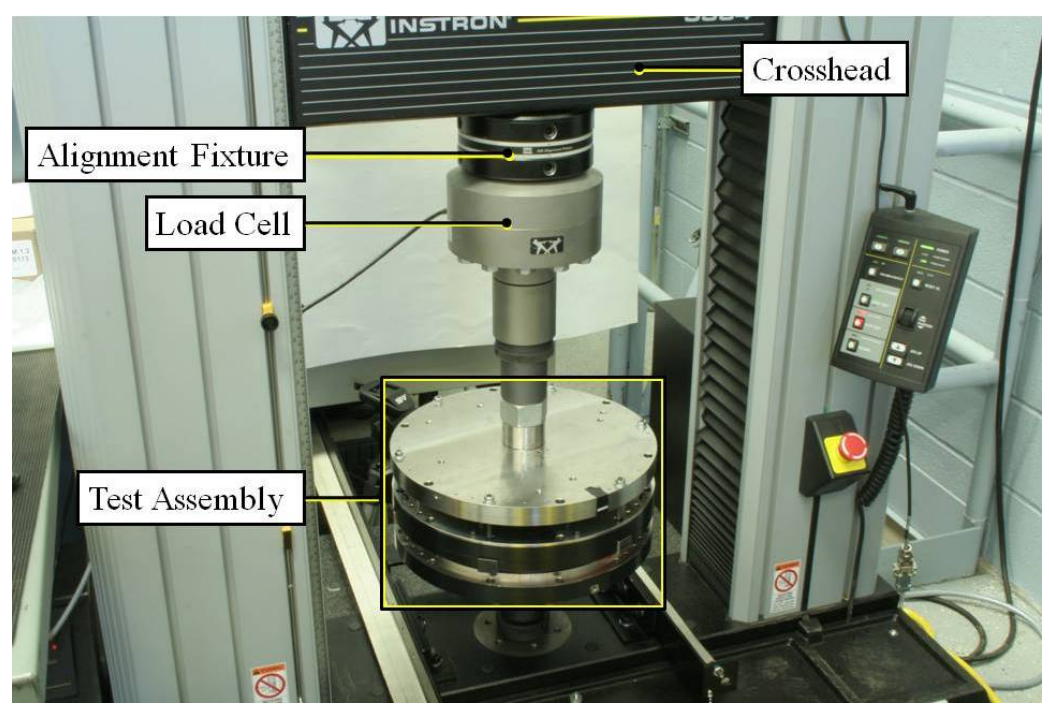

(a) Integrated components of the test rig

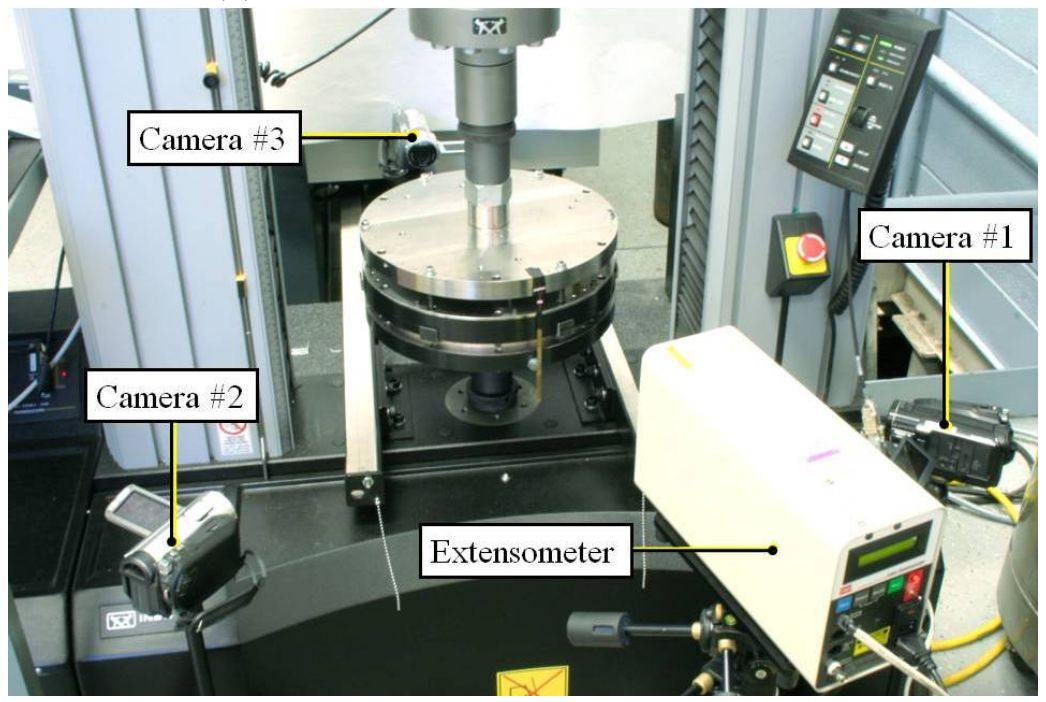

(b) External components used during testing

Figure 3.6: Photographs of the load frame and components used for composite seal bond strength testing 


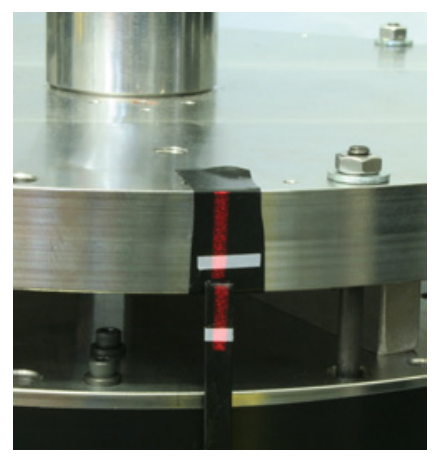

Figure 3.7: Reflective tape to measure interface separation with laser extensomer

Load is measured using a 33700 lbf capacity load cell with an accuracy of $\pm 0.29 \%$. A position encoder within the load frame measures displacement of the crosshead to an accuracy of \pm 0.001 inch. Displacement is also externally measured directly at the separating interface using an MTS Model LX 300 laser extensometer accurate to \pm 0.0001 inch.

The extensometer measures the relative displacement of the edges of reflective tape on the plates, as shown in Figure 3.7. The lower piece of reflective tape is on a thin metal rod that attaches to the bottom plate in order to reach the height of the laser area.

Video is recorded during the separation from different angles to aid analysis of the nature of failure for each test. Two high-definition and one DVD-quality camera (all are Sony HandyCams) are set up to maximize their utility during each test. For example, in tests on intentionally flawed specimens, at least one of the cameras is concentrated on the flawed region. On unflawed tests, the cameras are evenly spaced to capture the moment of failure wherever it may occur. The video record has proven to be an extremely useful tool in the understanding of the failure modes present in these tests.

An industrial Blue $M$ convection oven is used to accelerate the cure of the RTV adhesive in modest elevated temperatures and humidity. Although the RTV will cure at room temperature to adequate strength for most applications, it was found during the adhesive selection tests that curing the RTV in slightly elevated temperatures of 
$60-70^{\circ} \mathrm{C}\left(140-158^{\circ} \mathrm{F}\right)$ with an open container of water produced much stronger bonds in the RTV and as a result this process is incorporated into the seal test procedure.

\subsection{Experimental Procedure}

The test fixtures are designed to stress the bond in the seal without preemptively disturbing it prior to the moment of separation. Any premature pulling or twisting of the bulb could affect the results, and the procedure is developed with this in mind. The experimental procedure is comprised of three sub-procedures: RTV bonding, the pull-test, and the post-test procedure.

\subsubsection{RTV Bonding Sub-Procedure}

The RTV bonding process utilizes many of the lessons learned from the adhesive selection tests, especially in how to prepare the metal substrate surface and the technique for RTV application and cure. To prepare the rings (the metal substrate) for RTV bonding, they are first cleaned thoroughly with acetone. Then 150 grit sandpaper is used to remove any oxides or surface contamination and to slightly roughen the surface, which typically improves adhesion. Care is taken not to create deep scratches during sanding as this is not conducive to good bonding. The roughened metal substrate surface is then cleaned again thoroughly with acetone and left to dry. A thin layer of GE Silicones SS4004P primer is then applied to the area of the rings which will contact the RTV.

The elastomer surface must also be prepared for adhesive bonding with the RTV. First the seal is cleaned with acetone and sanded lightly with 150 grit sandpaper. The seal is blown off with compressed air to remove debris, then re-cleaned with acetone. At this point the seal bulb surface is prepared for adhesive bonding and the composite seal is bolted to the bottom plate.

Once the primer on the rings has air-dried, RTV is applied to the seal bulb and spread into the seal "valleys", as shown in Figure 3.8. A thin layer of RTV 
is also spread onto the appropriate surfaces on the rings to ensure a uniform film once compressed. The rings are placed in the seal valleys and the second plate is bolted to the rings. The upper plate and bottom plate are aligned with pins and bolted together to ensure no relative movement between the plates during cure and pull-test setup. At this point the bonding procedure is complete, and the assembly is placed in the oven to allow the RTV to cure for 5 days at $60-70^{\circ} \mathrm{C}\left(140-158^{\circ} \mathrm{F}\right)$ with a small pan of water.

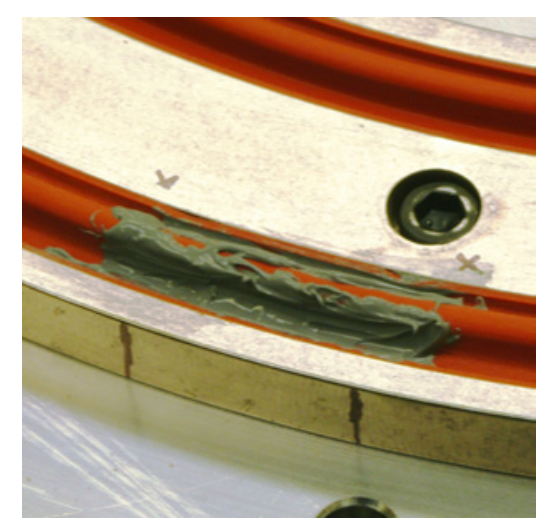

Figure 3.8: Photograph of RTV applied to seal bulb for a localized case pull-test

\subsubsection{Pull-Test Sub-Procedure}

Once the RTV has cured, the assembly is removed from the oven and attached to the lower rod of the load frame. The edges of the plates are covered with black tape to decrease glare in the video. A third plate, attached to the upper rod of the load frame, is aligned with the upper plate in the assembly using the load frame's alignment fixture. The top plate is lowered carefully until it contacts three spacers and is bolted to the middle plate. Then the lower and middle plates are detached using the six bolts that have held them together since the bonding procedure. This sequence is shown clearly in Figure 3.9. 


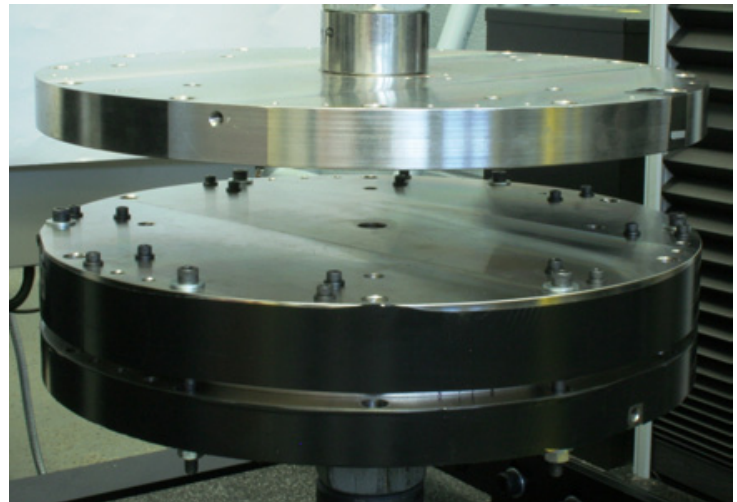

(a) Step 1: Assembly installed into load frame

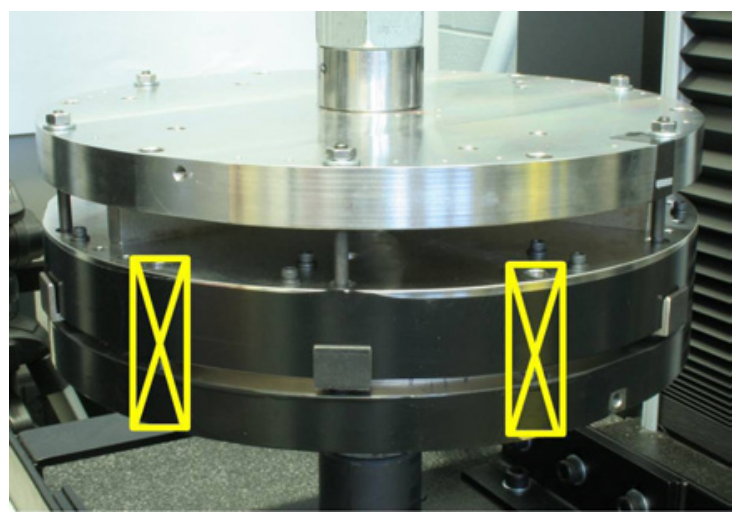

(c) Step 3 : Lower two plates unbolted

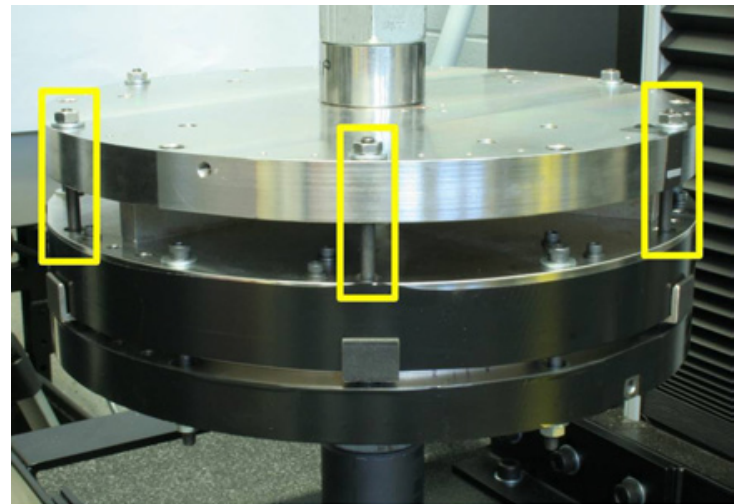

(b) Step 2: Upper two plates bolted together

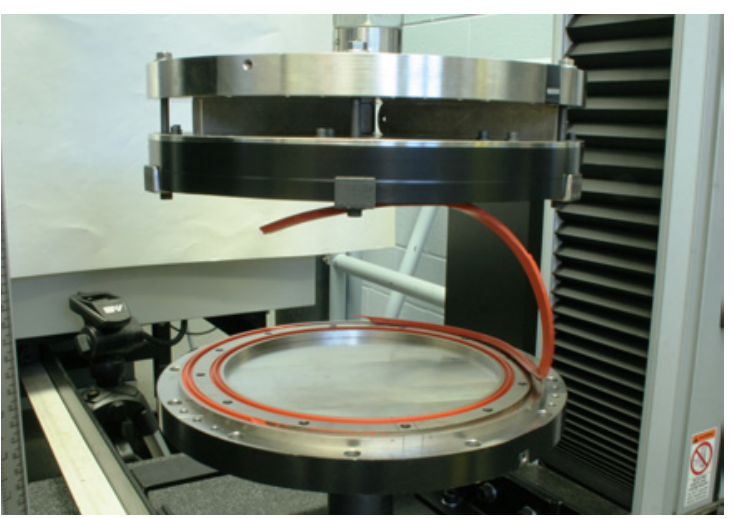

(d) Step 4: Interface separation

Figure 3.9: Photographs of the bond strength pull-test procedure

Once the components in the load frame are installed, the external devices are set up. The cameras are arranged and adjusted for the best picture of the regions of interest. The extensometer and its accompanying reflective tape pieces are set up at the proper distance and location.

The separation is rate controlled by the actuator of the load frame to match the expected displacement profile provided by the LIDS latching mechanism's motion. The separation begins slow and gradually increases, as shown in Figure 3.10. The chart also shows the measured profile from the position encoder and extensometer, revealing that the load frame successfully reproduces the expected separation profile that is input as a series of rate steps. 


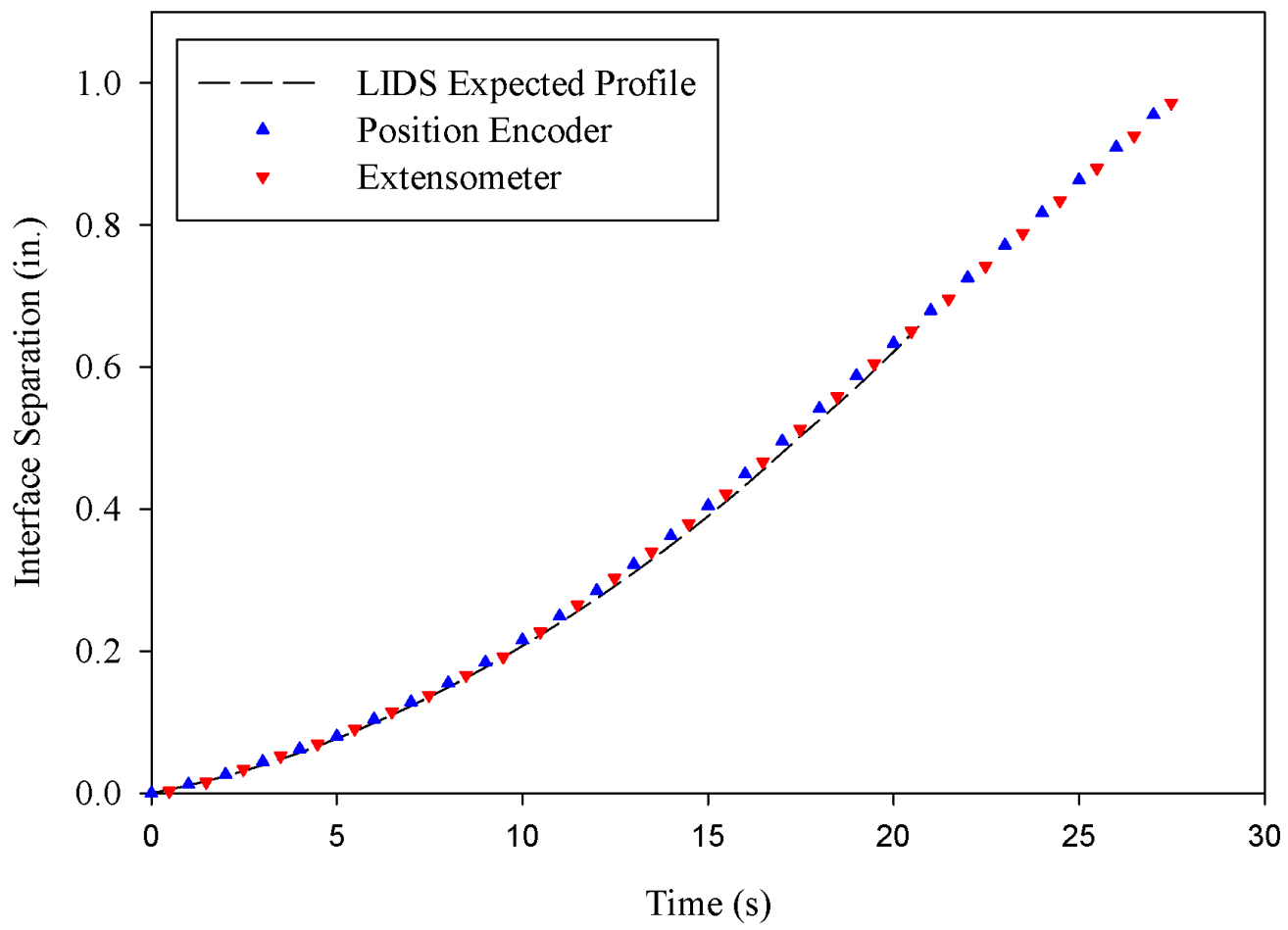

Figure 3.10: Graph of measured displacement vs expected LIDS interface separation profile

\subsubsection{Post-Test Sub-Procedure}

After the pull-test has been performed, the fixtures and specimens are removed from the load frame and any areas of interest are photographed. To allow the rings to be re-used, they are cleaned with acetone and a scrubbing pad. No foreign material is introduced to the rings that could inhibit surface preparation for RTV bonding for subsequent tests.

\section{References}

[1] C. C. Daniels, J. J. Oswald, M. B. Bastrzyk, I. Smith, P. H. Dunlap, Jr, and B. M. Steinetz. Experimental investigation of elastomer docking seal compression 
set, adhesion, and leakage. In AIAA SPACE 2007 Conference and Exposition, number AIAA 2007-6197, 2007.

[2] H. C. deGroh, III, S. K. R. Miller, I. Smith, C. C. Daniels, and B. M. Steinetz. Adhesion of silicone elastomer seals for NASA's Crew Exploration Vehicle. Technical Report NASA TM-2008-215433, National Aeronautics and Space Administration, 2008.

[3] American Society for Testing and Materials, West Conshohocken, PA. Standard Guide for Preparation of Metal Surfaces for Adhesive Bonding, 2008. 


\section{Chapter 4}

\section{Results and Discussion}

In order to understand more fully the parameter of bond strength in the composite seal design, a multifaceted approach is needed. Results of bond strength tests are reported from both a quantitative and qualitative perspective. The results are broken into the two loading cases, and each case includes both acquired data quantifying the bond strength and surface observations of the mode of failure. Both the numerical measurements taken during the pull-test and the subsequent qualitative observations provide valuable insight into the bond strength of a given seal specimen.

\subsection{Background}

On a microscopic level, the thin film of priming agent between the S0383-70 and eNi-plated aluminum surface plays a role in the chemical bonds formed in the interfacial region. However, the compositions of the priming agent and silicone compound are proprietary, and thus the molecular and chemical interactions in the primer-metal and primer-silicone regions are beyond the scope of this thesis. The focus of this work rests on a macroscopic level except where a microscopic view is revealing.

Like most elastomers, S0383-70 exhibits high nonlinear elastic elongation due to the molecular network of very long coiled polymer chains. The longer chains 
straighten as the material strains and eventually lead to abrupt rupture without much plastic yielding. This characteristic yields some interesting and complicated failure behaviors discussed in this chapter.

Further complicating the analysis is the geometry of the seal specimens. The separation load at the bond involves significant area in shear (groove side walls) and tensile loading (groove bottom). Upon separation, the bond at the groove bottom is being tested in tension while simultaneously the bond at the side walls is in shear. The net result is an increase in measured bond strength because shear is generally the stronger loading mode for bonds. On the top surface of the bulb, the stress profile is similarly complicated by the fact that the load imparted by the RTV adhesive to the top of the seal bulb is also a combination of shear and tensile components.

The thin layer of RTV adhesive can be described as a small compliant member between the rings and the seal bulb. This is analogous to a spring in series where some of the displacement measured is deformation of the RTV, not the seal bulb. Despite this, the relative thicknesses of the two components can lead to neglecting the RTV deformation.

The diameter of the seal specimens is large compared to the width of the bulbs and thus plane strain behavior can be assumed. This assumption allows for simpler comparison between different size bulbs by using linear quantities (e.g. lbf/inch of seal). However, once failure begins, the dimension along the circumference is involved, and hence comparisons using the same linear quantities are not possible.

The analysis could be simplified by using basic test articles defined by various standards such as ASTM Standard Test Methods D429 ${ }^{1}$ or D794². This would limit the bondline load to uniaxial tension or linear peel, rather than the more complicated groove geometry of the seal specimens. However, there are several marked advantages to testing fabricated seals as discussed in detail in Section 2.3. Several representative comparisons with previously reported adhesion results on similar seal designs are given here. These tests are performed by compressing the seal against a metal plate, holding for a specified amount of time, and then separating the interface according 
to the expected LIDS separation profile.

\subsection{Cohesive vs. Adhesive Failure}

Adhesion is the attraction between two different substances because of the intermolecular forces between the materials. ${ }^{3}$ Cohesion describes the attractive forces between molecules within a single substance. ${ }^{3}$ At a bonded interface of two different materials, bond failure can occur in two distinct ways differentiated by location. Cohesive failure occurs in one of the two materials near the interface. This is the desirable failure mode for an engineered bonded joint because it indicates that the bond achieved with a material is stronger than the material itself.

The second type of bond failure is adhesive failure in the interfacial layer between the bonded materials, indicating that the bond is in fact the weaker region. Both cohesion and adhesion are functions of local conditions, meaning some areas can fail cohesively and others adhesively on the same test specimen.

For the seal bonds tested, both adhesive and cohesive failure are observed at the seal-to-retainer bond, and cohesive failure in the seal and in the RTV are observed at the RTV-to-seal bond. In addition, failure in the seal away from either bond is defined here as material failure, although in a broader sense it is a variation of cohesive failure. The five failure modes are represented by letters, as shown in Figure 4.1. The quality of a bond is often rated using the percentage of bond area pertaining to each failure mode. For example, the most desirable rating is $100 \%$ cohesive failure in the silicone, whereas a $100 \%$ adhesive failure is the least desirable. The variability in percentage of area for each failure mode is due to material imperfections, voids, surface contaminants, and a variety of other possibilities. Figures 4.2 and 4.3 show the representative portions of area that exhibited each failure mode on each test specimen. Table 4.1 gives the exact values represented in the figures. 


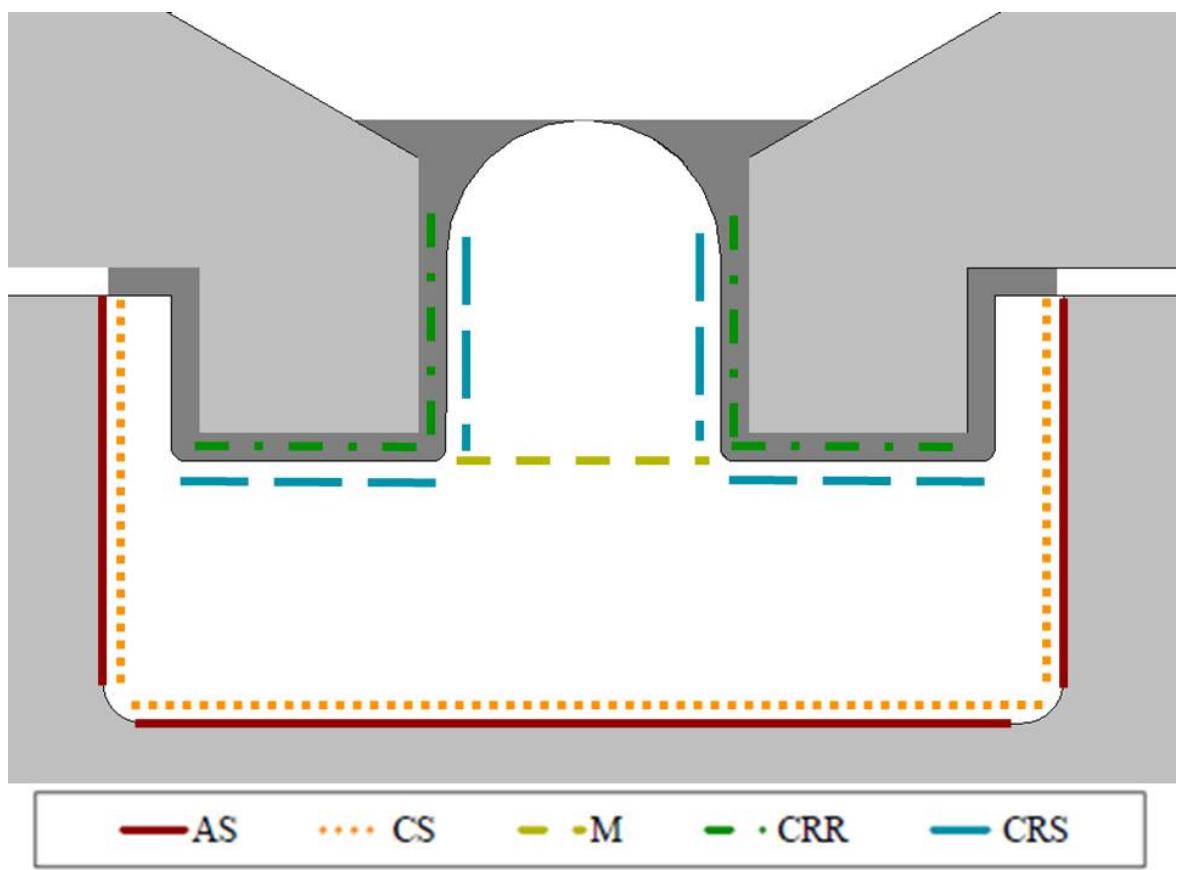

Figure 4.1: Schematic showing the location of the 5 failure modes observed. The letter designations and colors correspond to the bond ratings given in Figures 4.2 and 4.3 

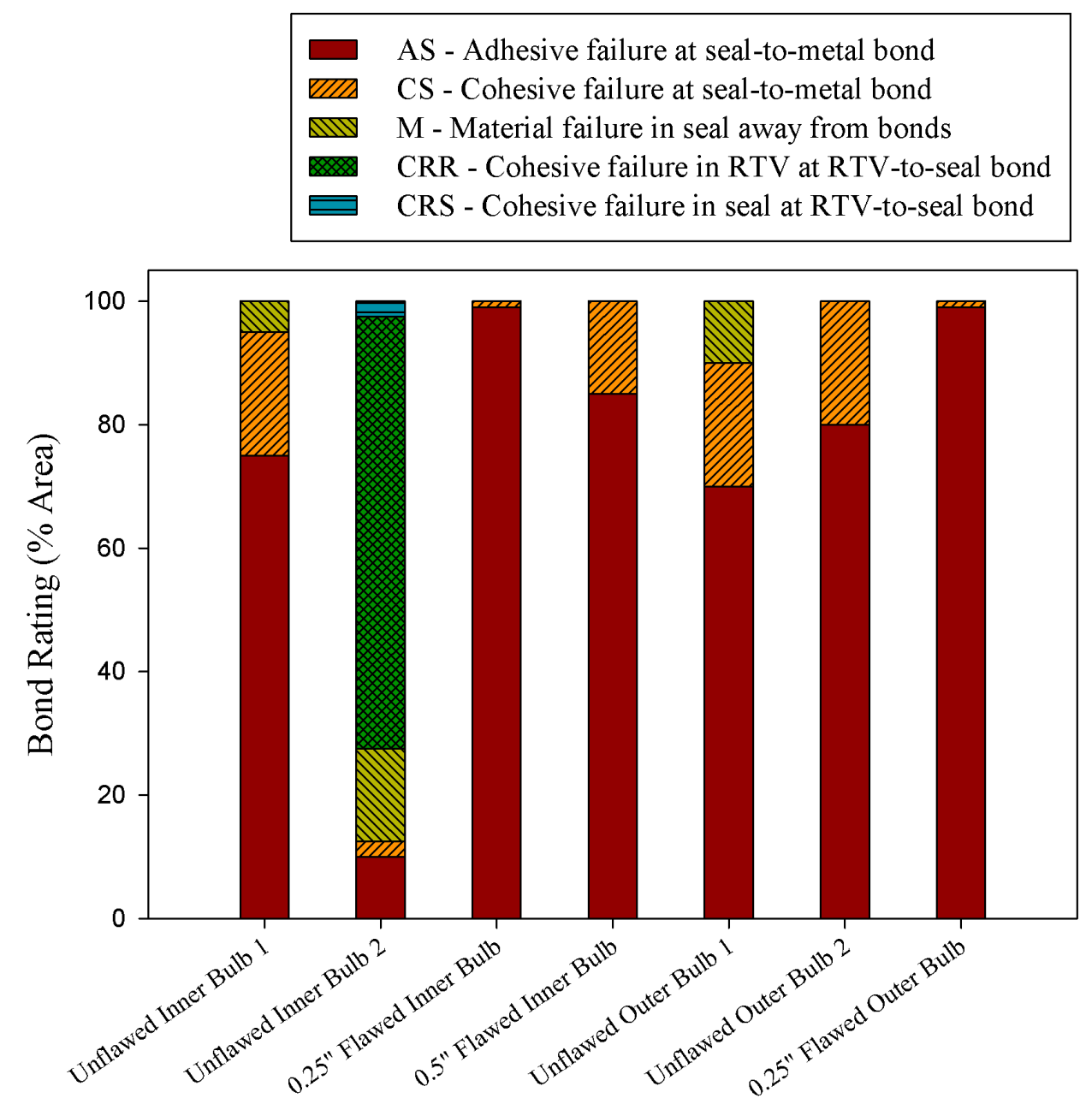

Uniform Adhesion Case Test Specimens

Figure 4.2: Bar chart summarizing the bond ratings of all uniform adhesion case test specimens 


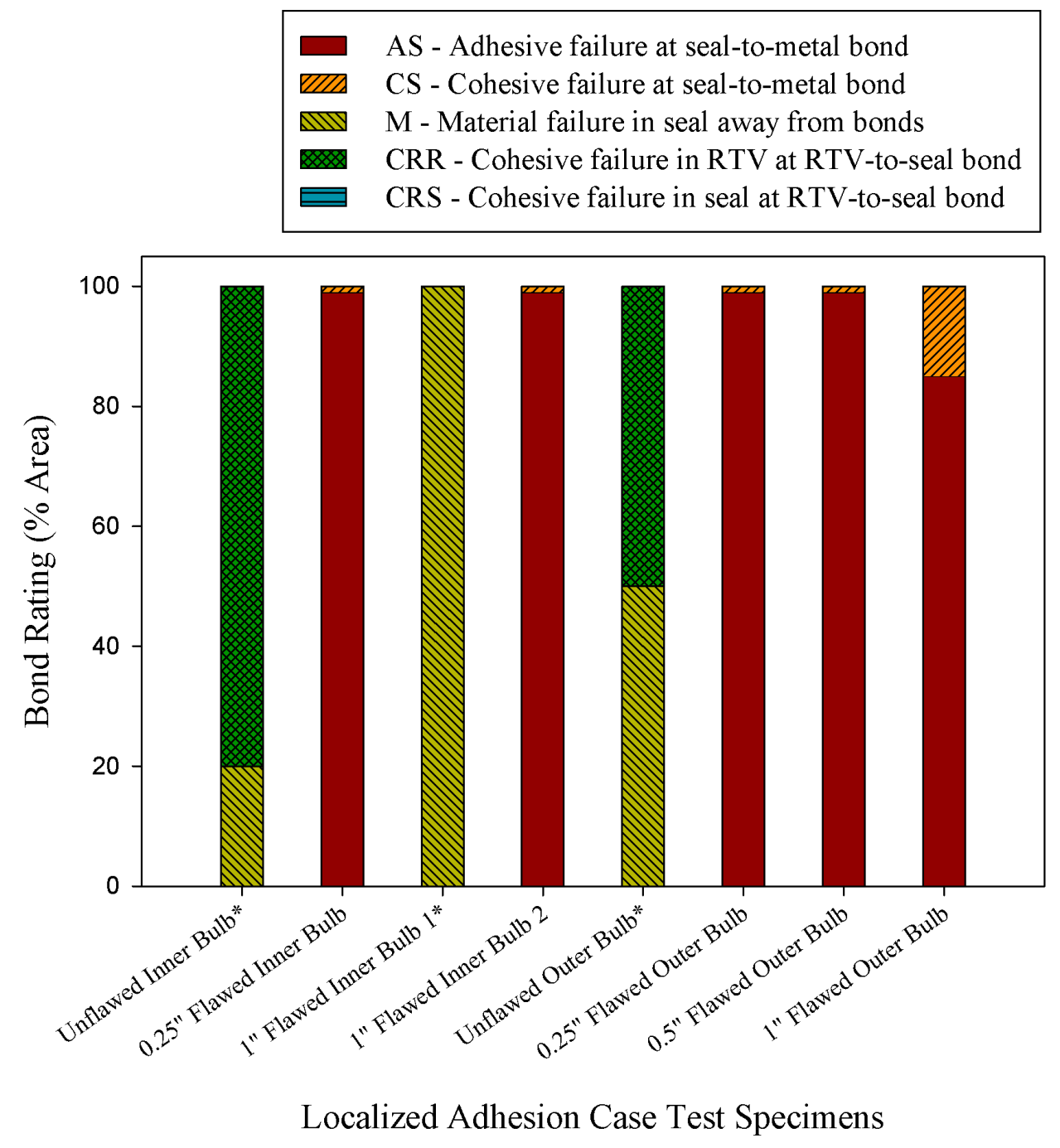

Figure 4.3: Bar chart summarizing the bond ratings of all localized adhesion case test specimens

All seal samples tested show a mixture of cohesive and adhesive failure to differing degrees as well as other additional modes. In tests where the silicone-to-metal bond failed, there is a wide scatter of the relative portions of each failure mode. In several tests, the RTV-to-seal bond failed. In adhesive or cohesive failure of strictly the RTV, nothing is learned about the seal performance as the only information produced is the strength of the adhesive used. However, cohesive failure of the seal bulb at the RTVto-seal interface is revealing because it indicates the possibility of adhesion "skinning" 


\begin{tabular}{|c|c|c|c|c|c|c|c|c|}
\hline \multirow{9}{*}{$\begin{array}{l}\text { Uniform } \\
\text { Adhesion } \\
\text { Case }\end{array}$} & \multirow[t]{2}{*}{ Test No. } & \multirow[t]{2}{*}{ Bulb } & \multirow{2}{*}{$\begin{array}{l}\text { Flaw Length } \\
\text { (in.) }\end{array}$} & \multicolumn{5}{|c|}{ Bond Rating } \\
\hline & & & & $\mathrm{AS}$ & CS & $\mathrm{M}$ & CRR & CRS \\
\hline & 1 & Inner & 0 & 75 & 20 & 5 & & \\
\hline & 2 & Inner & 0 & 10 & 2.5 & 15 & 70 & 2.5 \\
\hline & 3 & Inner & 0.25 & 99 & 1 & & & \\
\hline & 4 & Inner & 0.5 & 85 & 15 & & & \\
\hline & 5 & Outer & 0 & 70 & 20 & 10 & & \\
\hline & 6 & Outer & 0 & 80 & 20 & & & \\
\hline & 7 & Outer & 0.25 & 99 & 1 & & & \\
\hline \multirow{8}{*}{$\begin{array}{l}\text { Localized } \\
\text { Adhesion } \\
\text { Case }\end{array}$} & 1* $^{*}$ & "Inner & "0 & & & 20 & 80 & \\
\hline & 2 & Inner & 0.25 & 99 & 1 & & & \\
\hline & $3^{*}$ & Inner & 1 & & & 100 & & \\
\hline & 4 & Inner & 1 & 99 & 1 & & & \\
\hline & $5^{*}$ & Outer & 0 & & & 50 & 50 & \\
\hline & 6 & Outer & 0.25 & 99 & 1 & & & \\
\hline & 7 & Outer & 0.50 & 99 & 1 & & & \\
\hline & 8 & Outer & 1 & 85 & & 15 & & \\
\hline
\end{tabular}

*Partial failure. Ratings are percentage of area involved in failure.

Table 4.1: Summary of exact bond ratings as represented in Figures 4.2 and 4.3

the seal bulb upon separation. This would result in silicone residue on the mating surface and is a serious, if less severe concern compared to some of the other observed failure modes. In addition to cohesive and adhesive failure, material failure of the silicone away from the interface is observed.

The results for each loading case are divided into categories signifying the dominant observed failure mode, but all observed modes are discussed. Tests which produced substantial levels of cohesive failure are classified as cohesive failure mode dominant although more total groove area exhibited adhesion failure. In several of the cohesive mode dominated tests, the groove side walls did in fact produce a larger area in cohesive rather than adhesive failure though the groove bottom did not. Other tests resulting in no clear dominant mode are classified as mixed. 


\subsection{Uniform Adhesion Case}

The uniform adhesion case is a simulation of an extreme adhesive load being generated by compressing S0383-70 silicone against a metal surface. This loading case implicitly assumes homogeneity on the entire surface of both materials, and in idealized nominal operation, this is a valid assumption on the full-scale LIDS interface. On orbit, the flange surface will be exposed to months or years of the space environment, so there should not be any directional preference of AO, UV radiation, or ionizing radiation effects on the mating surface. The seal bulb will also be exposed to the space environment prior to docking, and the effects of that exposure also should be uniform around the circumference.

During nominal operation there is a possibility of a thermal gradient from one half of the flange or seal circumference to the other. This could occur if docking is performed when the axis of the docking port lies perpendicular to the path of solar

radiation. The half of the interface facing the sun would be warmer than the half facing deep space. The assumption of uniform adhesion is still reasonable in this case because half of the sealing interface circumference is still large in relation to the groove geometry. One half of the seal showing increased adhesion due to thermal effects would not concentrate the stress on the seal bond much more than uniform adhesion over the entire surface.

The assumption of uniform adhesion at the sealing surface breaks down with the realistic consideration of localized events such as surface contamination, MMOD damage, and mechanical wear. These scenarios are discussed in Section 4.4.

\subsubsection{Cohesive Failure Mode}

\section{Fracture Surface Observations}

Figure 4.4 shows a representative area of significant cohesive failure in the bottom of the groove on an unflawed seal specimen. For this particular section of area, it is not immediately clear whether cohesive or adhesive failure constitutes more of the 
bond. The groove bottom is covered in red silicone residue in what appears to be patterns along the circumference. This could be due to priming agent being brushed in this direction, leading to distributed spots of superior bond formation and resulting in cohesive failure.

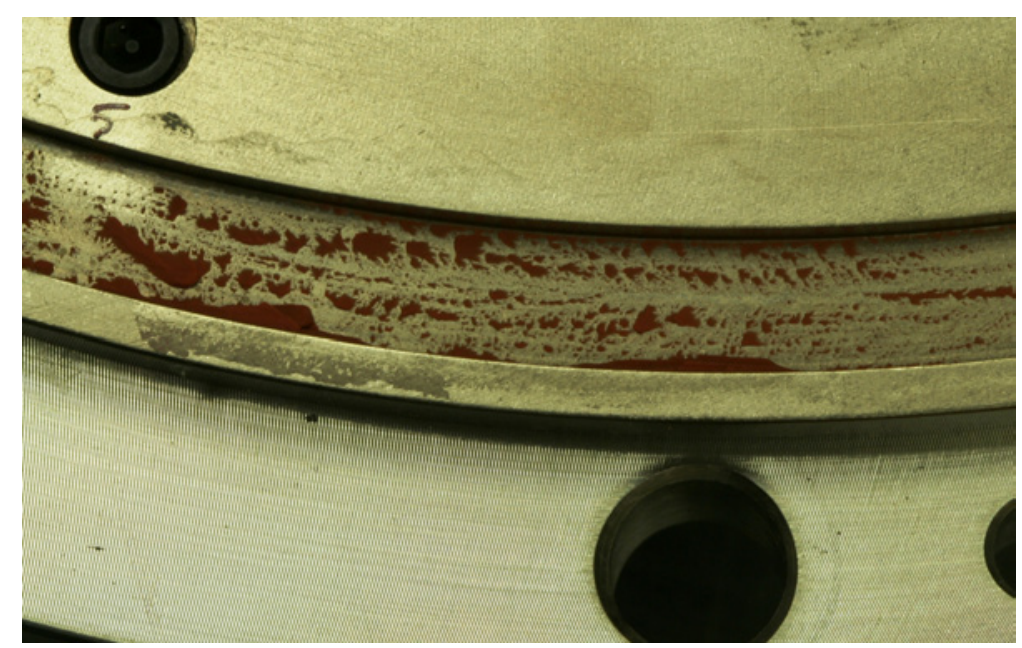

Figure 4.4: Photograph of a fracture surface showing significant cohesive failure (CS), along with adhesive failure (AS)

Fracture surfaces were examined with an optical microscope to see more detail about the relative amount of cohesive failure. Figure 4.5 shows a representative area of significant cohesive failure under 7.1X magnification. As in Figure 4.4, there appear to be linear patterns of silicone residue along the groove. 


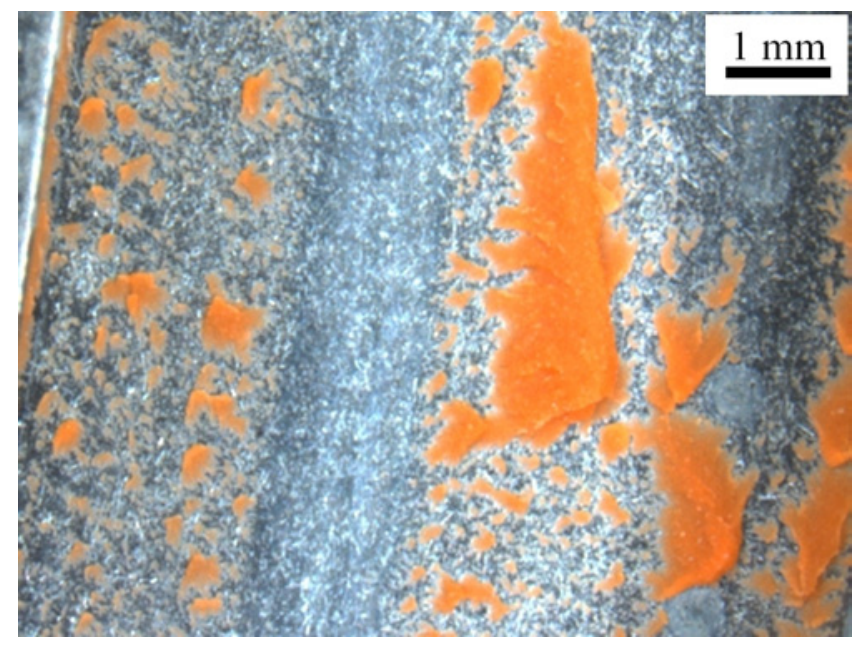

Figure 4.5: Optical micrograph of fracture surface under 7.1X magnification showing cohesive failure (CS) patterns

Under higher magnification (63X), areas which appear initially as sparse spots of residue are actually interspersed with fields of much smaller residue spots with size on the order of $100 \mu \mathrm{m}$ (Figure 4.6). Thus, the area of cohesive failure is more than that visible with the naked eye.

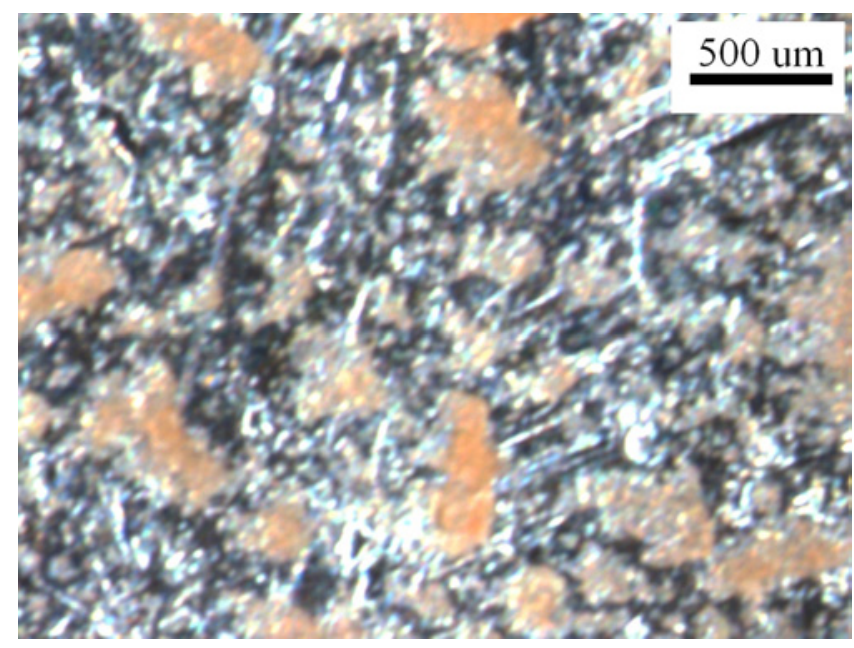

Figure 4.6: Optical micrograph of fracture surface under 63X magnification showing small spots of cohesive failure (CS) invisible to the naked eye 


\section{Data Analysis}

The load-displacement data of the outer bulb test shown in Figure 4.4 is given in Figure 4.7 along with the inner bulb test on the same specimen. This test produced the highest measured load of the uniform adhesion test series, with the peak measured load on the outer bulb test at $4579 \pm 7 \%$ lbf. The uncertainty associated with the measured load in all tests is outlined in Appendix A. As seen in Figure 4.7, the loaddisplacement curves of the inner and outer tests agree closely up until the moment of rupture in the inner test at $3842 \pm 6 \%$ lbf. For both curves, there are distinct regions of behavior. In the first 0.030 in. of separation there is an approximately linear relationship between load and deflection. At the initiation of rupture, there is a flattening of the curve as small areas begin to fail and can no longer carry load.

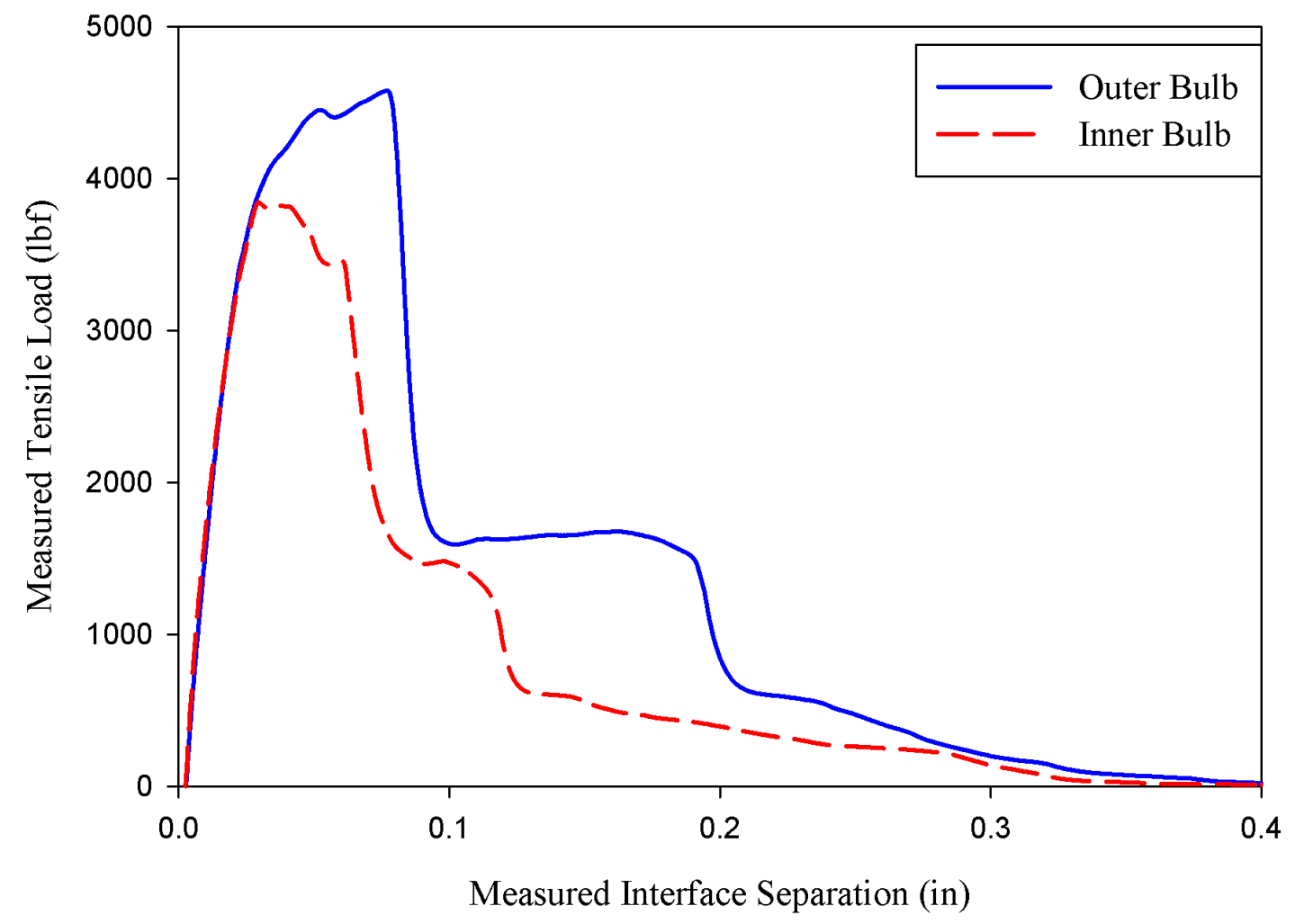

Figure 4.7: Graph of measured results for uniform load case exhibiting cohesive failure 
The first steep drop in load represents the initial bond failure in part of the seal. In the initial linear region, the tensile load is shared by all of the bond area distributed around the circumference of the seal and hence the resultant force vector acts axisymmetrically at the center of the seal. However, once a portion of the bond fails, the load is then carried only at the remaining area which shifts the net force vector off-center, resulting in a bending moment on the interface. This bending moment is enough to deflect the load frame rods for a brief period. This is visible in the video as a slight shifting of the plates and is observable in the data as the second flat plateau, from 0.1 to 0.2 in. on the outer bulb test. Although visible, it is less pronounced on the inner bulb curve. The second steep drop indicates that most of the remaining bonded area has failed. The final slightly downward sloping region indicates the last remaining sections of silicone that are stretching and tearing away from the retainer.

A useful quantity to compare between tests on the different size bulbs is the load at fracture per linear inch of circumference. This value can be easily used to estimate full-scale parameters. Figure 4.8 reveals that the results of the four uniform adhesion case tests producing significant adhesion are much less variable on a linear basis. 


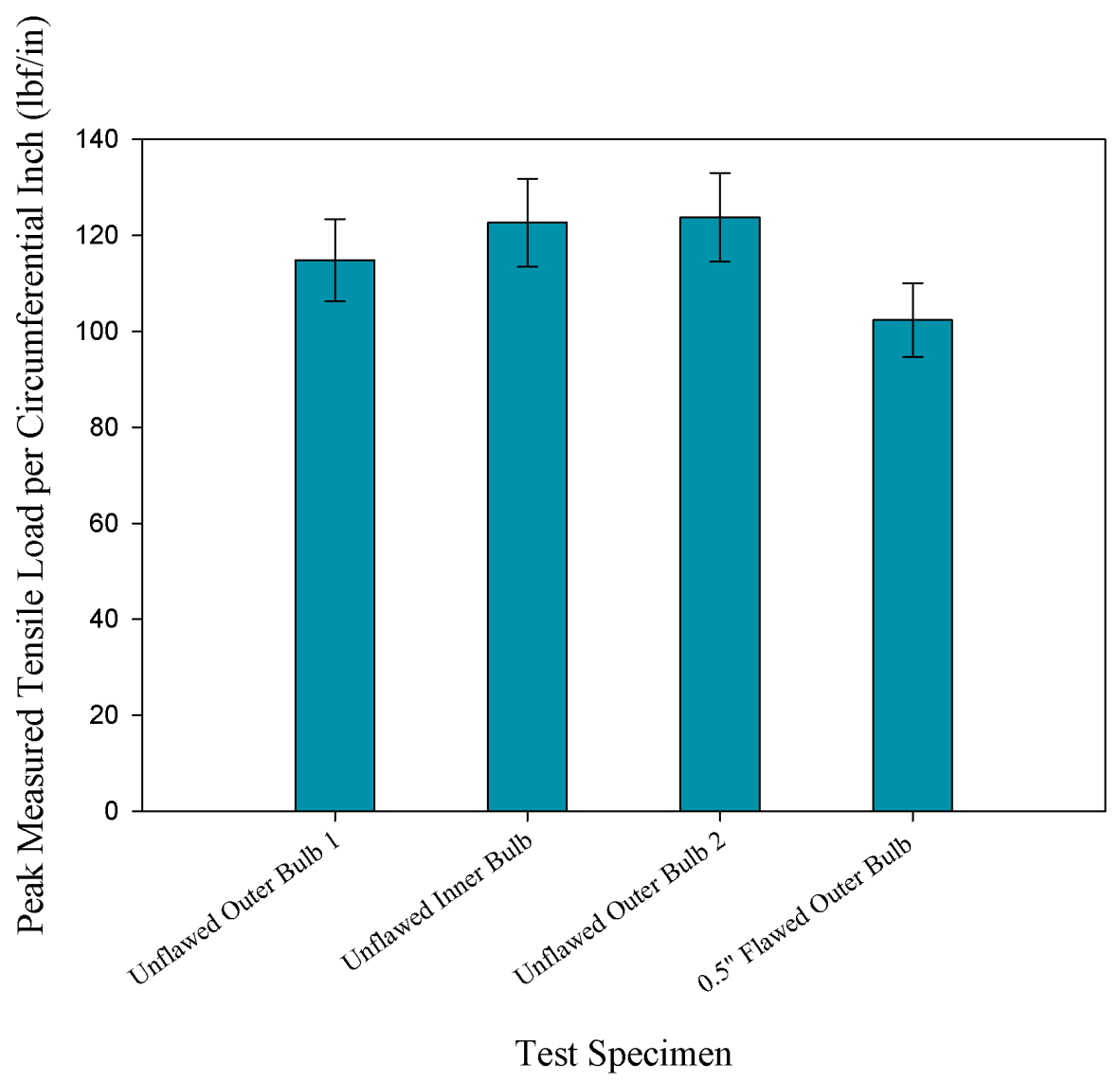

Figure 4.8: Bar chart representing failure load per circumferential inch of a single seal bulb

There is no correlation between flaw length and the load produced or the observed failure mode. The unflawed seal specimens generally show higher load measurements and more cohesive failure, but the load to fracture is not increased by larger flaws. It was originally anticipated that disbond locations would be the initiation points of rapid peel out of the seal bulb. The explanation for this lies in the mechanics of separation. As the interface separates, the short section of disbonded seal bulb arcs upwards and begins to stretch. Eventually, enough of the tension in the seal bulb will concentrate at the silicone-metal bond line to commence peel-out. However, this behavior is not observed in the tests because failure somewhere in the bonded area initiates at a displacement less than that required for lifting and stretching the 
unbonded section of the bulb. As a result, the initial bond failure occurs in an arbitrary location and not necessarily at the disbond.

In the results discussed above, the final region of stretching and failure of remaining segments of the seal can be seen in the video, as shown in Figure 4.9. The elastomer appears white under the high elongation prior to failure.

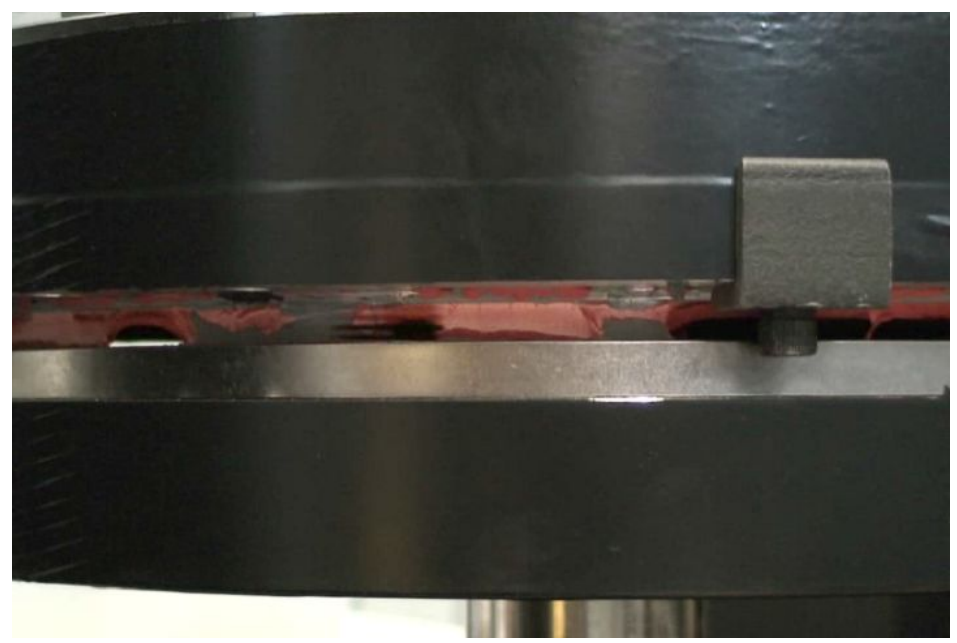

Figure 4.9: Video frame just prior to final elastomer failure. The white sections are highly elongated regions of elastomer

\subsubsection{Adhesive Failure Mode}

\section{Fracture Surface Observations}

The only mode to dominate overwhelmingly in the uniform adhesion case was adhesive failure. Both seal bulbs tested on a seal with 0.25 " flaws displayed greater than $99 \%$ adhesive failure. A representative area of the groove surface, shown in Figure 4.10, is entirely absent of any silicone residue. 


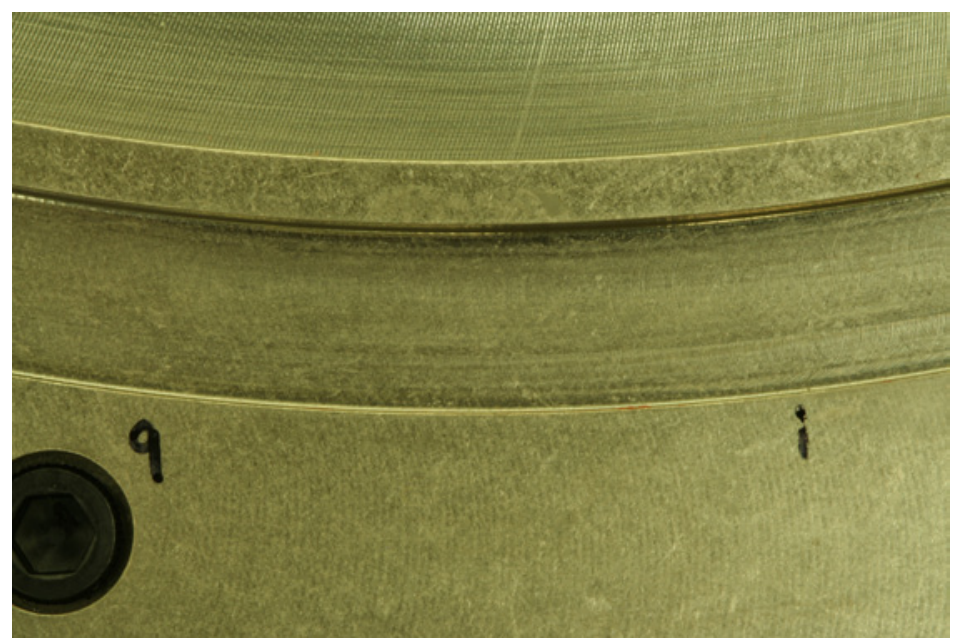

Figure 4.10: Photograph of a representative area showing exclusively adhesive failure (AS)

Under magnification, there are still no visible traces of elastomer on the adhesively failed surface. Figure 4.11 shows a representative area of adhesive failure under 12.5X magnification.

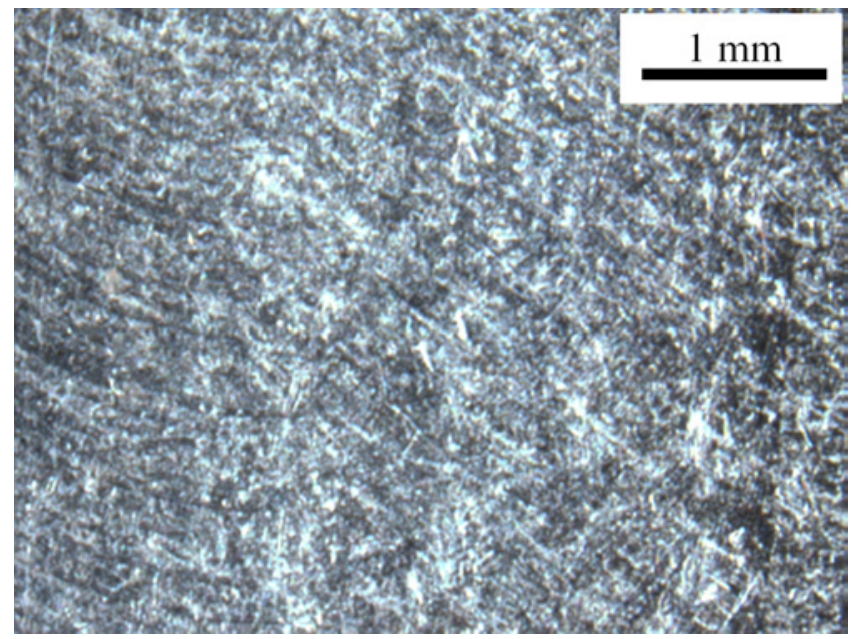

Figure 4.11: Optical micrograph of adhesive failure (AS) surface at 12.5X magnification. No visible elastomer residue is present 


\section{Data Analysis}

Figure 4.12 shows the load-displacement curves of the two uniform adhesion case tests that failed almost exclusively in the adhesive mode. The outer bulb curve follows a similar pattern as that seen in Figure 4.7, indicating that a bond which fails adhesively can still be strong enough to deflect the load frame rods once the net force vector is off-centered. This is an important observation: Although the measured load is relatively high, the observed mode of failure is undesirable.

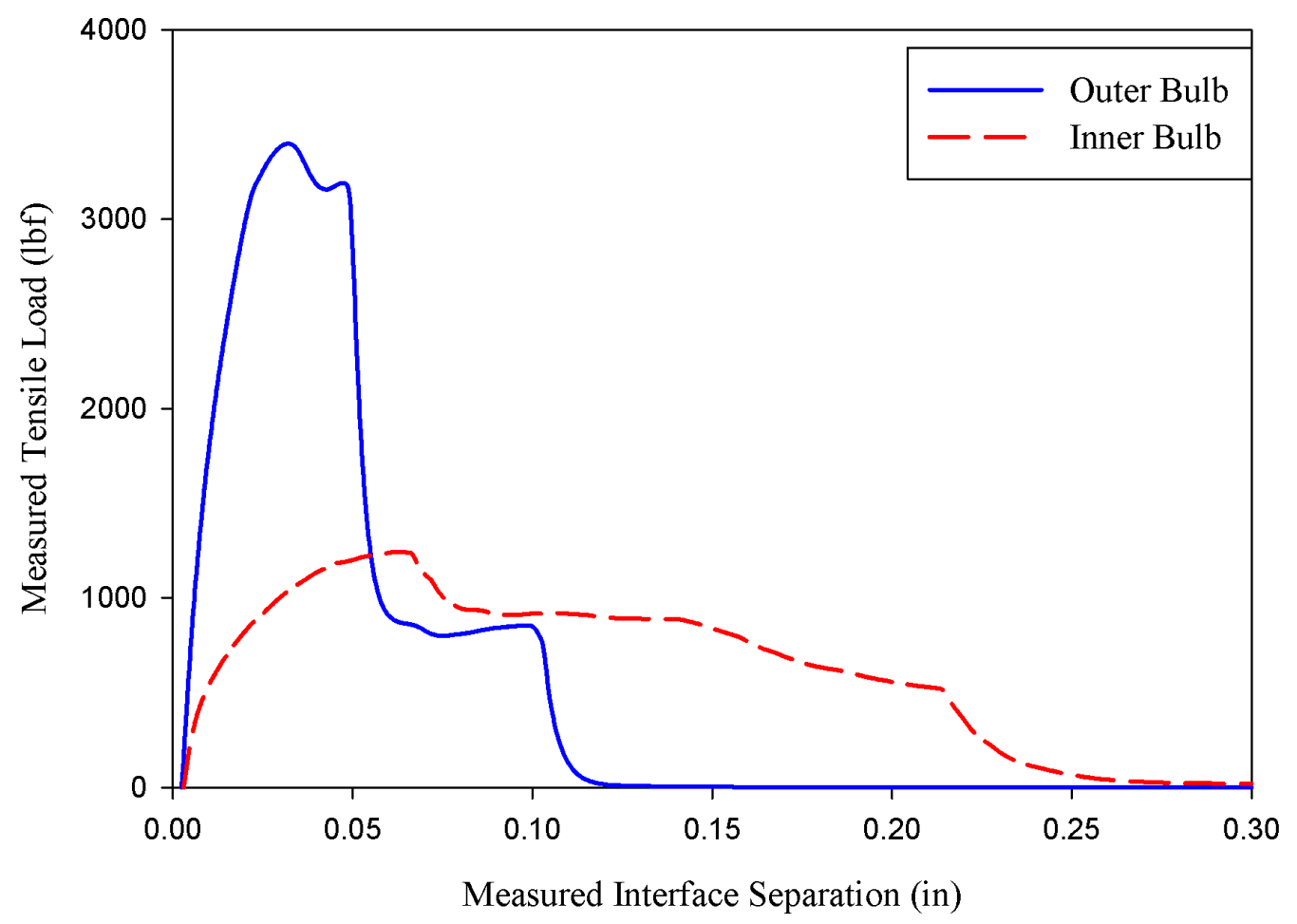

Figure 4.12: Graph of measured results for uniform load case exhibiting overwhelmingly adhesive failure

The inner bulb curve in Figure 4.12 does not follow the expected pattern due to the nonuniformity of the bond failure. In this inner bulb test, a portion of the circumference failed at the RTV-to-seal interface as shown in Figure 4.13. The RTV 
failure is characterized by a lower peak load measured, followed by a long slow decline in measured load as the remaining length of seal bulb is peeled out. Also, the RTV failure probably began occurring in what otherwise would have been the linear region of the curve, resulting in the decreasing stiffness curve shown in Figure 4.12 from 0 to 0.06 in.

An observer looking only at the measured load in the inner bulb test in Figure 4.12 might come to an incorrect conclusion about the bond strength without knowing how the failure took place. The nature of failure tells as much about the strength of the bond as the measured force at the point of failure.

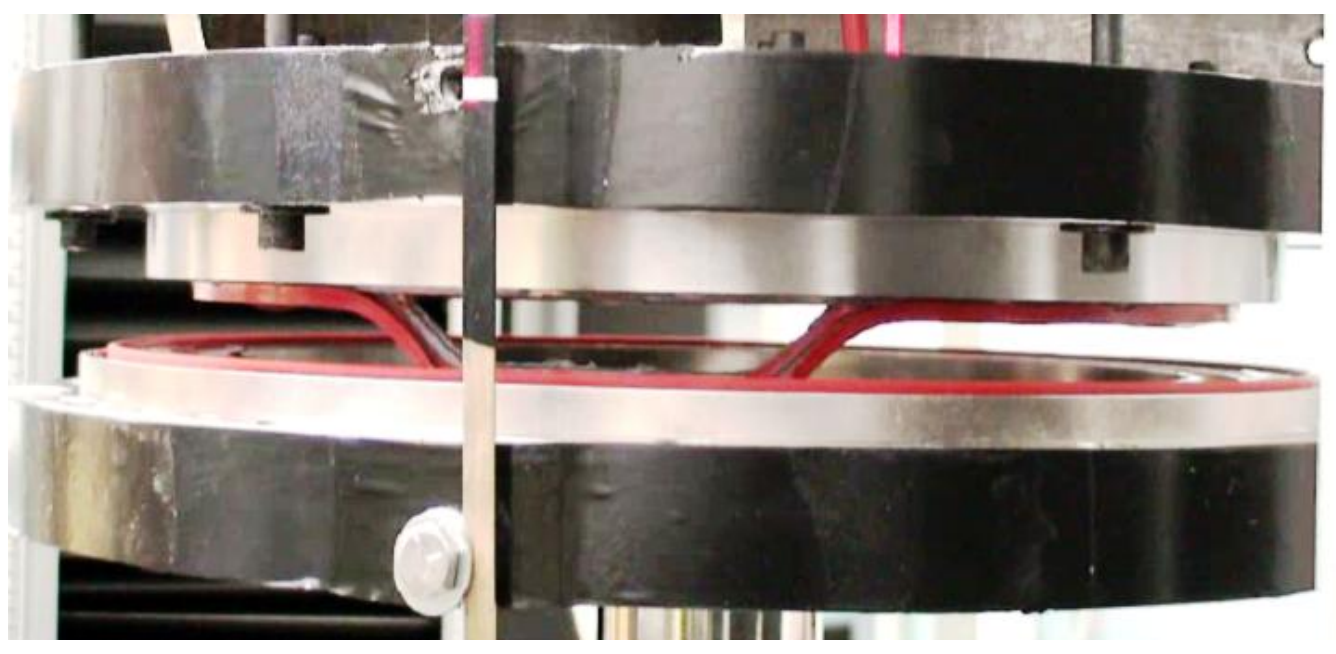

Figure 4.13: Video frame of the partial RTV failure in a uniform adhesion case leading to an altered load-displacement curve

\subsubsection{Mixed Failure Mode Fracture Surface Observations}

The remaining uniform adhesion test result is the separation of an unflawed inner seal bulb that exhibited multiple failure modes. The dominant mode of failure for this test is cohesion in the RTV at the RTV-to-seal bond (60\%), but the other modes observed include material failure, adhesive and cohesive failure at the groove bottom, and cohesive failure in the seal material at the RTV-to-seal bond. The quantities of 
these modes are given in the bond rating found in Table 4.1.

Figures 4.14 and 4.15 show the areas of different failure modes. The important observation that can be made from this test is that the silicone-to-metal bond can vary widely in strength, even around the circumference on the same seal bulb. In this photograph, the outer groove is also visible, and it clearly exhibits mostly adhesive failure with sparse cohesive failure areas. Therefore, the bond strength can vary widely between different seal bulbs on the same composite seal, as well as different areas of the same bulb.

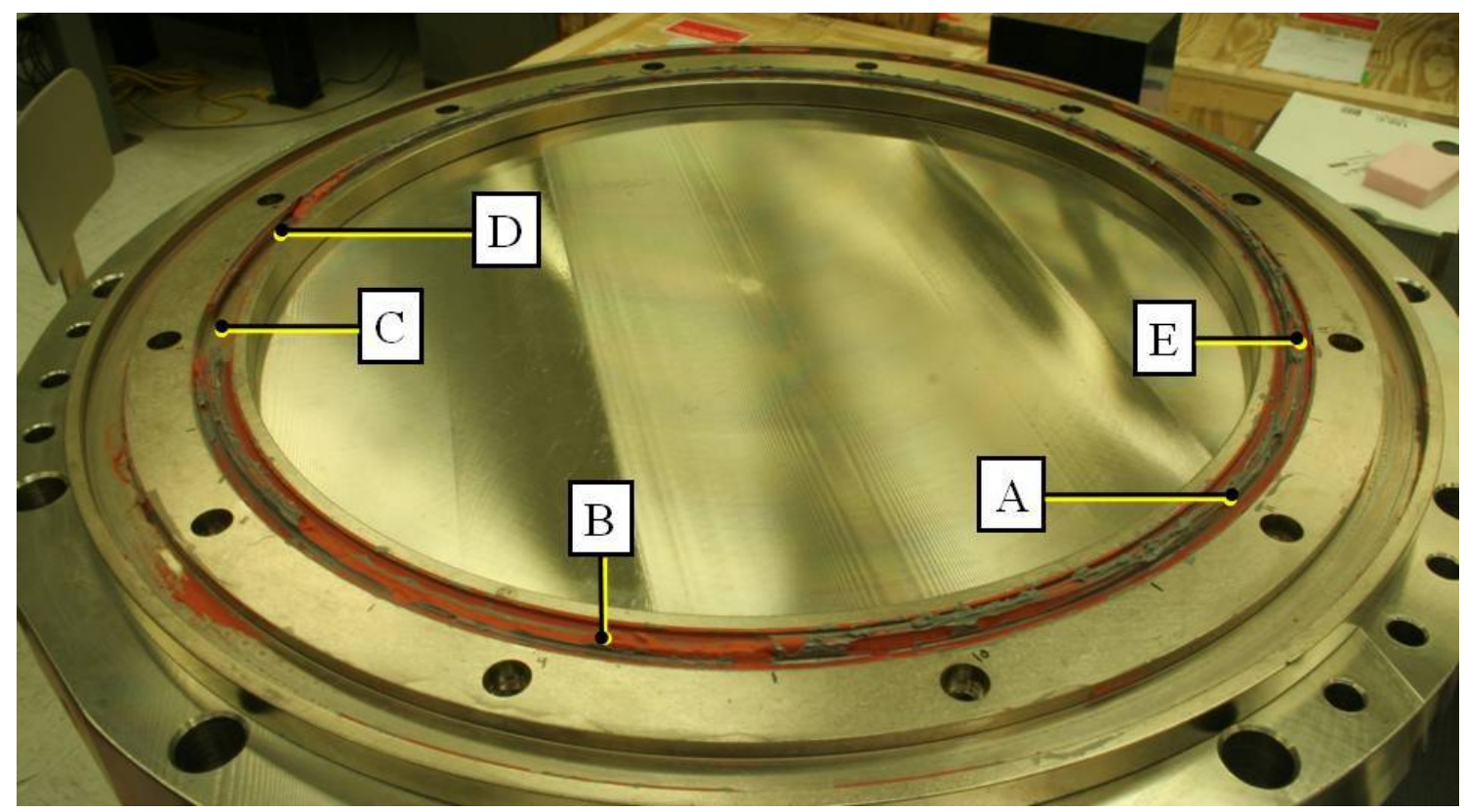

Figure 4.14: Photograph of the seal showing multiple failure modes. Closeup views of each area are given in Figure 4.15 


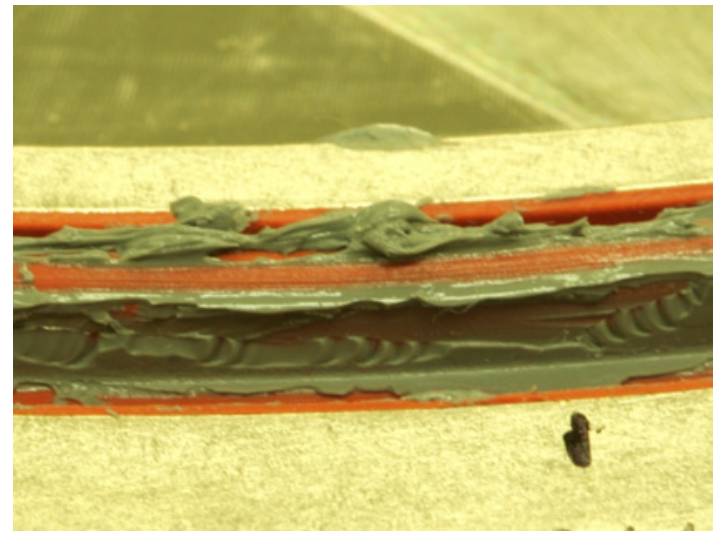

(a) RTV-to-seal cohesive failure (CRR)

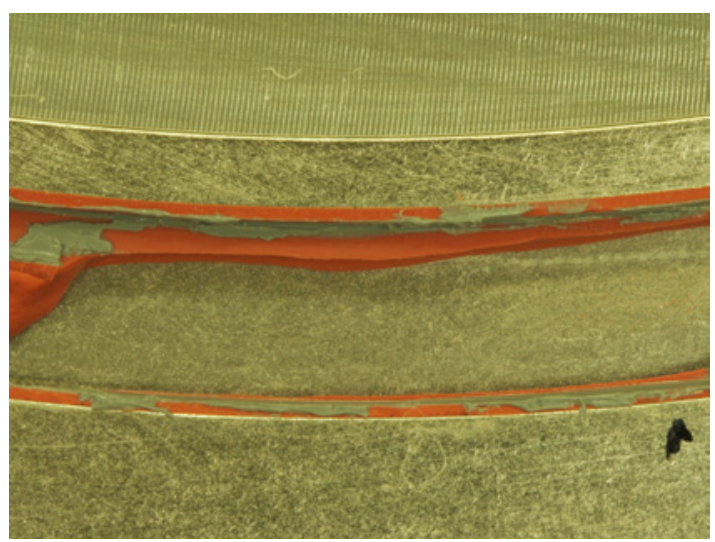

(c) Silicone-to-metal adhesive failure (AS)

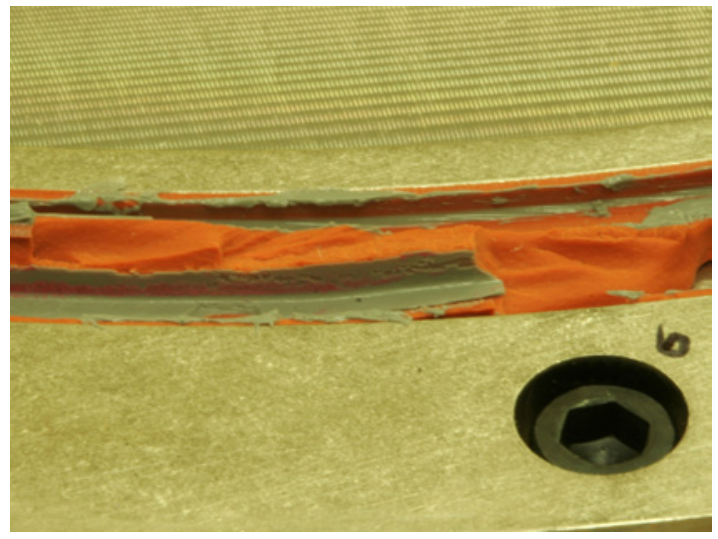

(b) S0383-70 material failure (M)

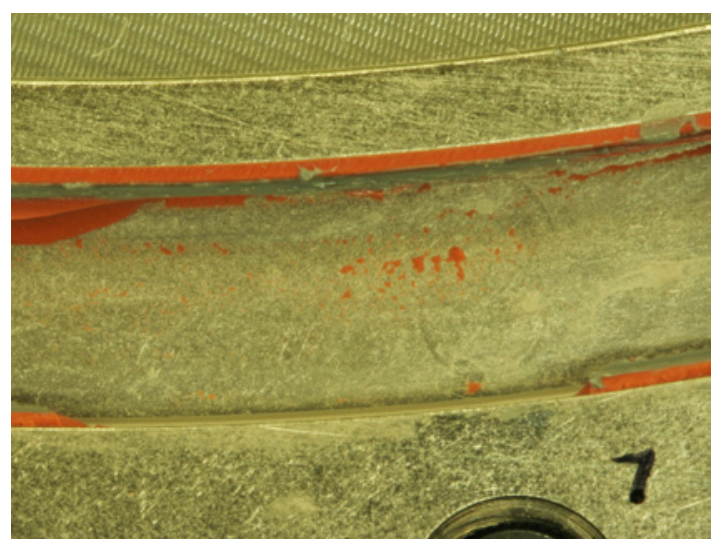

(d) Silicone-to-metal cohesive failure (CS)

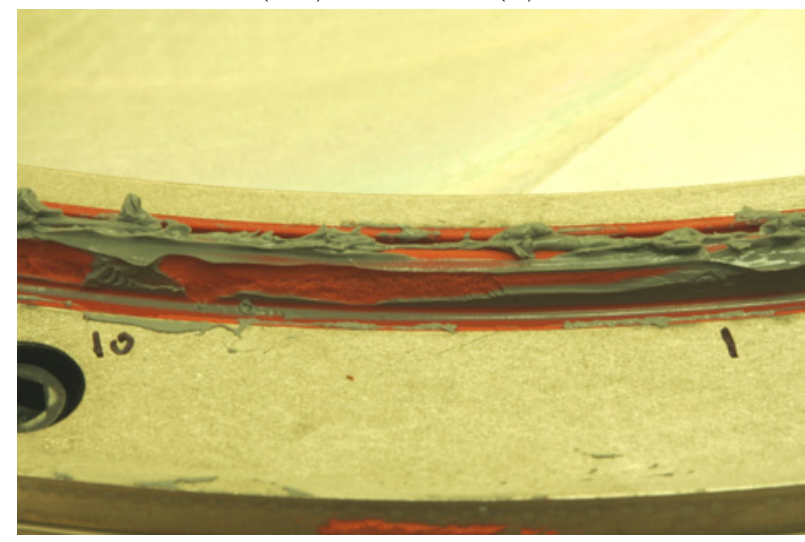

(e) Cohesive failure in the S0383-70 at the RTV bond (CRS)

Figure 4.15: Photographs of fracture surfaces in a test showing mixed failure modes 


\section{Data Analysis}

The data collected during the mixed failure mode test is given in Figure 4.16. The video of this test when compared to the data suggest that the plateaus seen around 0.1 and 0.3 in. are due to parts of the seal releasing. A large section releases at a time corresponding to the steep drop in load and no shifting of the plates is visible.

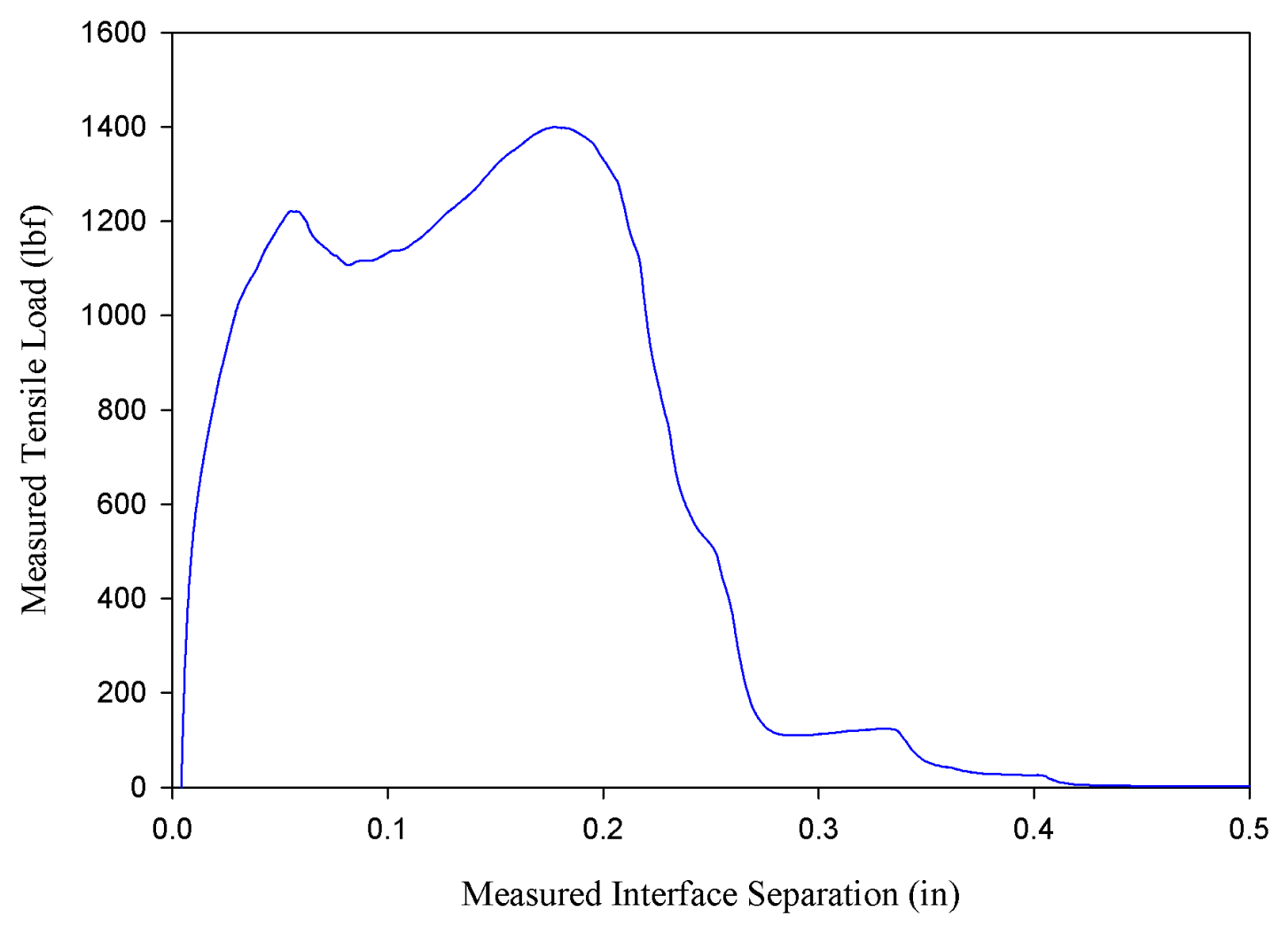

Figure 4.16: Graph of measured results for uniform load case exhibiting mixed failure modes

\subsubsection{Comparison to Seal Adhesion Data}

Comparisons to experimentally measured adhesive loads help to form quantitative acceptance criterion for the composite seal design. However, the results presented in this chapter can be misleading if only the net measured load is considered. For ex- 
ample, several tests show high fracture loads, but subsequently displayed undesirable failure modes such as complete adhesion. Yet, clearly some metric must be used to accept the seal design without doing destructive tests on every fabricated seal, and comparison with other adhesion data is an obvious choice.

Of the two bond strength loading cases tested, uniform adhesion is the more appropriate mode for comparison with adhesion tests of seals. The adhesion tests are performed by compressing a seal against a metal plate for a specified amount of time, and then separating it while measuring load. The manner in which these tests are run intentionally minimizes the amount of surface contamination possible on the sealing surfaces. The seals are stored in closed containers and the plates are stored in cabinets, decreasing the risk of scratches or other localized events. Prior to testing, the sealing surfaces are cleaned as part of the test process. Therefore, the adhesion demonstrated in these tests is distributed more or less uniformly on the sealing surfaces.

The peak loads measured on two representative adhesion tests are compared to the peak loads measured in tests in the three failure modes of the uniform adhesion case in Figure 4.17. The values reported are load per circumferential inch of one seal bulb. The lowest measured value is the mixed failure mode test at $44.70 \pm 6 \%$ lbf, greater than the adhesion tests listed by a factor of approximately 47 and 93 for the CBM and EDU58 geometries, respectively. It should be stressed that these are representative tests only, not averaged values. The testing of these particular seal samples is ongoing. 


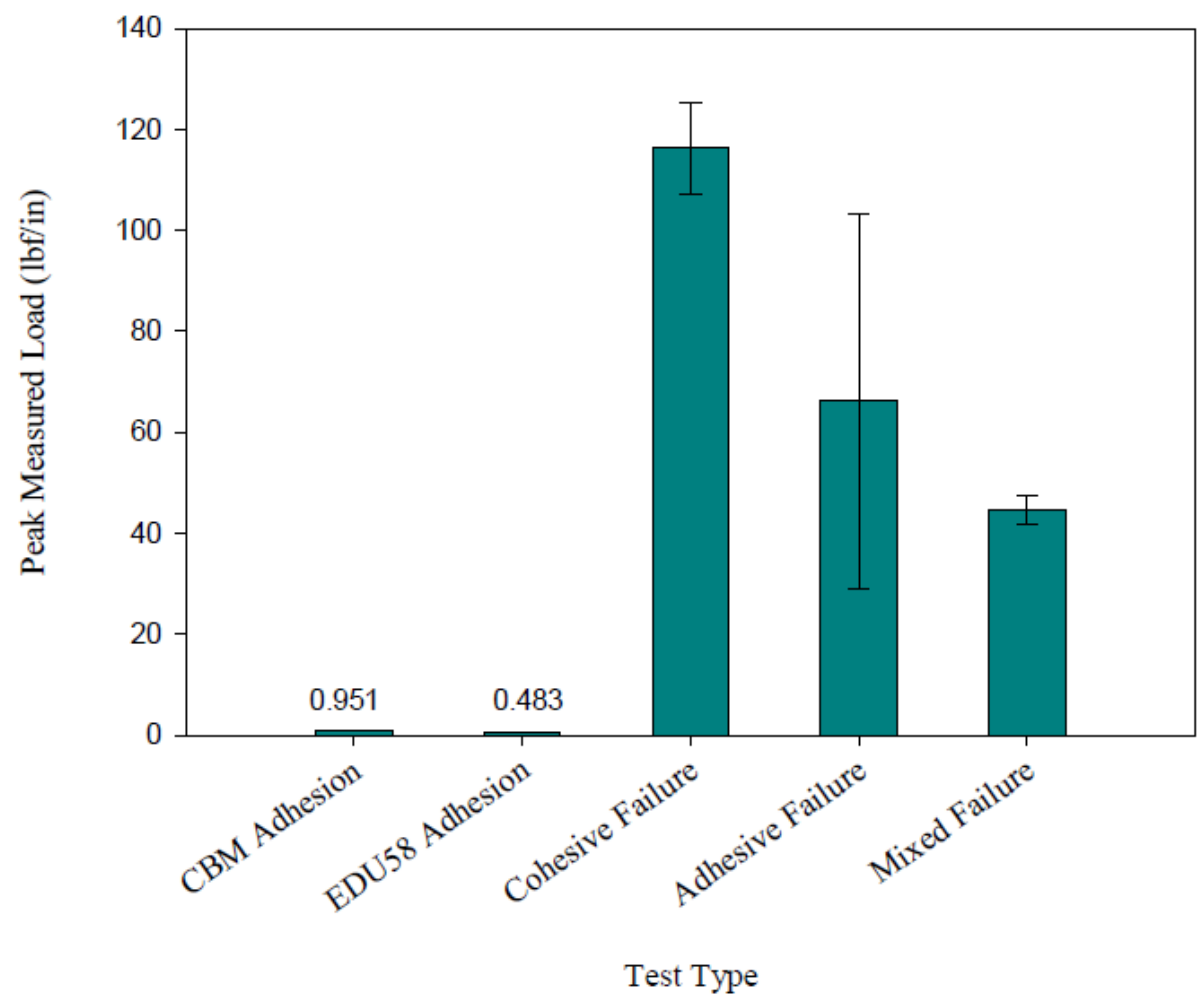

Figure 4.17: Bar chart comparison of peak measured loads on adhesion tests and on bond strength tests

\subsection{Localized Adhesion Case}

The localized adhesion case can be viewed as the worst-case scenario for the seal bond as it represents increased localized adhesion coinciding precisely with an area of complete disbond. Although improbable, the Progress incident exposes the possibility of increased localized adhesive activity and its effects. The primary potential for increased localized adhesion stems from surface contamination of the interface and material variation in the elastomer. As surface contamination would likely be nonuniform, specific areas would have chemical conditions more conducive to adhesion with the silicone. Less probable singular events that could lead to increased adhesive activity include a MMOD impact and mechanical wear due to cycled docking and undocking. Neither of these events have been investigated in relation to increased 
adhesion, they are merely proposed as ways in which fresh, possibly more adhesive material could be revealed on orbit.

\subsubsection{Adhesive Failure Mode}

\section{Fracture Surface Observations}

Five of the eight seal bulbs tested in the localized adhesion case produced predominantly adhesive failure. The tests in this category detached from the seal retainer by way of slow, steady peel out along the entire circumference, resulting in the complete removal of the seal bulb in one piece, as shown in Figure 4.18. The bottom of the grooves in these tests exhibited the same conditions as the adhesive failure mode tests of the uniform adhesion case, and Figure 4.10 is an accurate representation for

reference. Inspection of the groove side walls on several tests revealed small spots of apparent cohesive failure as well. 


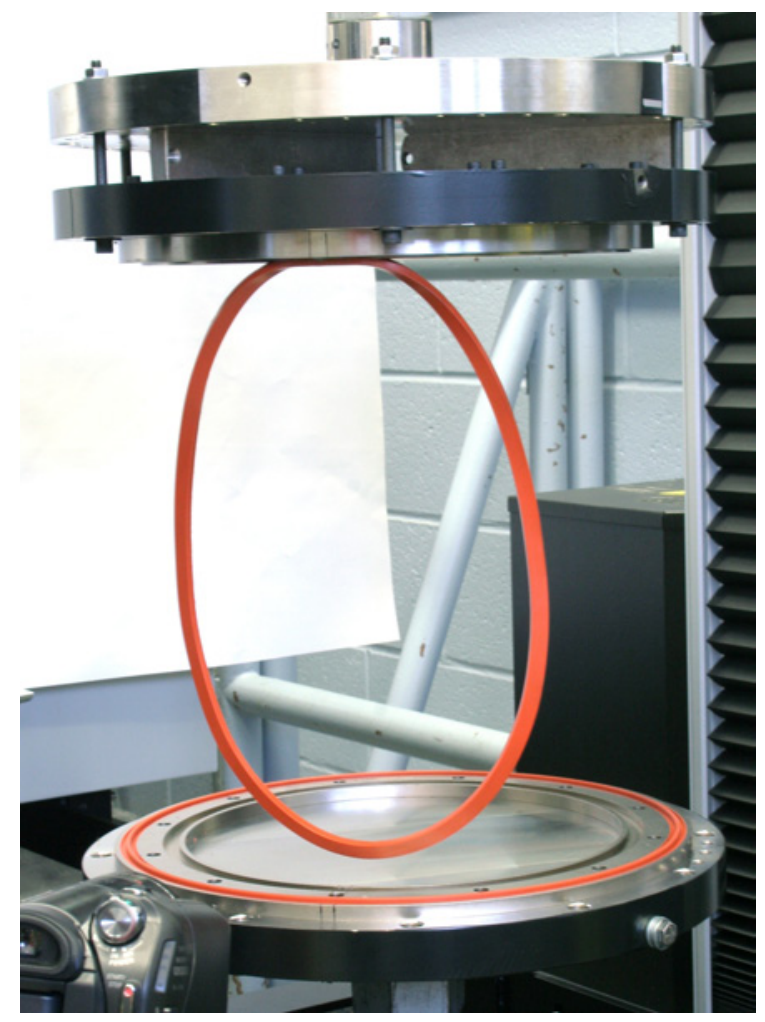

Figure 4.18: Photograph of the complete removal of the seal bulb in one piece due to localized adhesion

\section{Data Analysis}

The load-displacement curves for these tests are given in Figure 4.19. There is not a clear correlation between the size of the flaw and the force required to peel the bulb out of the groove. Because the RTV is applied to 1 inch of the circumference at the disbond location, one would expect a higher load to be required in order to rupture the remaining bonded 0.75 in. on a 0.25 in. flawed seal than the remaining 0.5 in. on a 0.5 in. flawed seal, for example. Although the 1 in. flawed seal in Figure 4.19 displays the lowest measured peak load, the other curves do not show this relationship. 


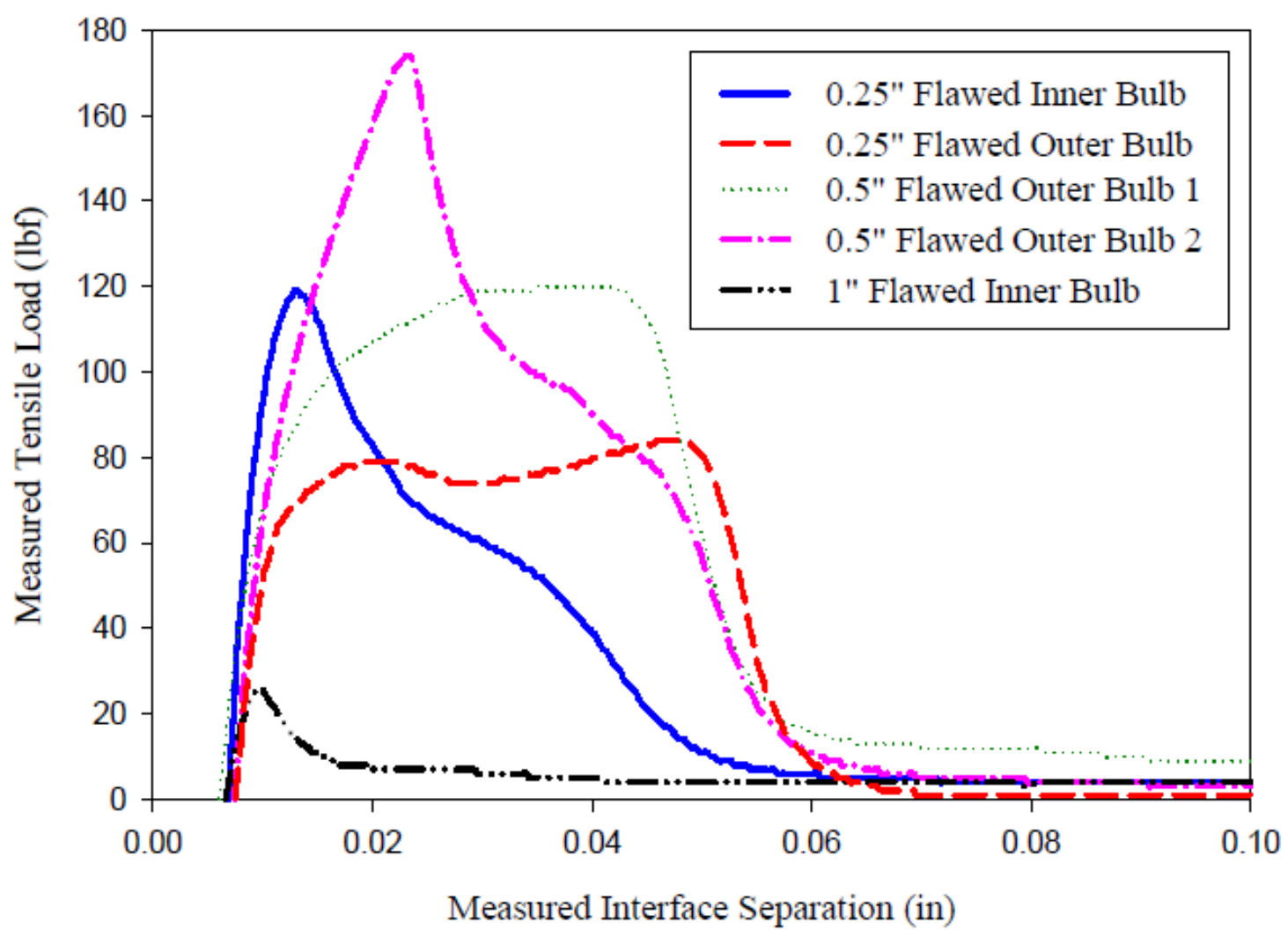

Figure 4.19: Graph of measured results for localized adhesion case exhibiting adhesive failure

Similar to the uniform adhesion case tests showing cohesive failure, there are distinct regions of the curves, which can be analyzed with the help of video footage of the interface during separation. There is a nearly linear elastic region at initial separation which lasts for as little as the first 0.010 in. for the $1 \mathrm{in}$. flawed specimen. At the initiation of failure, two general shapes are observed in Figure 4.19.

The $0.25 \mathrm{in}$. flawed inner bulb, $0.5 \mathrm{in}$. flawed outer bulb 2, and $1 \mathrm{in.}$ flawed inner bulb curves display a sharp peak followed by a gradual downward slope until approximately $0.06 \mathrm{in}$. It is difficult to determine from studying the video what is occurring during the initial sharp peaks, because they occur within the first $0.030 \mathrm{in}$. of separation, preventing a view with the camera. 
The curves for the 0.25 in. flawed outer bulb and 0.5 in. flawed outer bulb 1 tests show a gradual plateau followed by a steep drop in load at approximately 0.05 in. of separation. It is clear from both curve shapes and the available video that peeling of the bulb begins at 0.06 in. as all data sets except the 1 in. case converge at that value to the near-constant peel loads. The 1 in. case clearly begins peel much earlier as expected, as the length of the adhesive area is the same as that of the disbonded area.

As the bulb peels out along the circumference, the tension in the bulb is reacted by the still-bonded area. However, because of the angle of peel, the stress in the stillbonded region is concentrated at the peel line. This is visible as a faint white line along the edge of the bulb still in the groove, as seen in Figure 4.20. The concentration of stress at the peel line leads to the very low load values necessary for peel out once it has initiated.

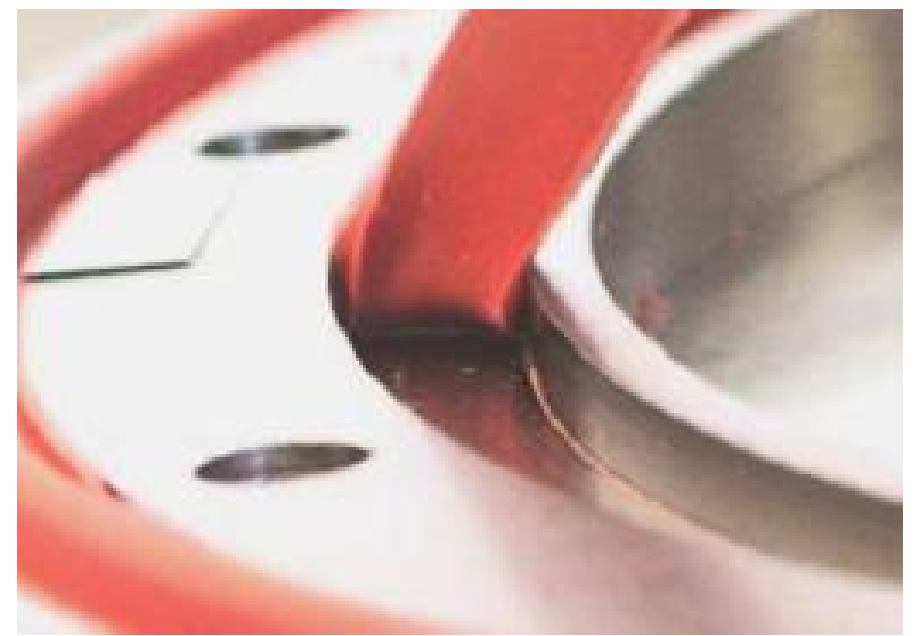

Figure 4.20: Video frame of the white peel line just visible at the base of the seal bulb when it separates from the groove bottom

\subsubsection{Material Failure Mode Fracture Surface Observations}


The remaining tests done under the localized adhesion case failed in the seal material rather than at the bond. For this worst-case type of loading, this is the more desirable failure mode of the two observed because it prevents the large seal bulb from being loosely attached at only one point. In the material failure mode, small silicone fragments could be left behind on the flange, impeding the subsequent docking process. However, if the fragments were small enough, it is feasible that some level of seal could be achieved, although the redundancy provided by two seal bulbs would be lost. In a situation where the entire seal bulb peeled out of the groove, it would most likely lie across the interface, preventing any kind of seal from being attainable. In addition, a full-scale LIDS seal could tangle in other components near the interface if it was only attached at a single point on the circumference.

The material failures observed in this category exhibit several interesting features. Although the failure is often seen to follow regions and lines of geometric stress concentration, it does not always do so. As an example, Figure 4.21 is an optical micrograph of the region of failure near the edge of the seal crown. As expected, the failure originates along a straight line visible in the middle of the image at the corner created by the seal crown and valley. The upper left corner of the image shows that the failure traveled across the crown base and then propagated at nearly a right angle downward into the bulb. The complex molecular network through which the failure travels leads to such unpredictable behavior. 


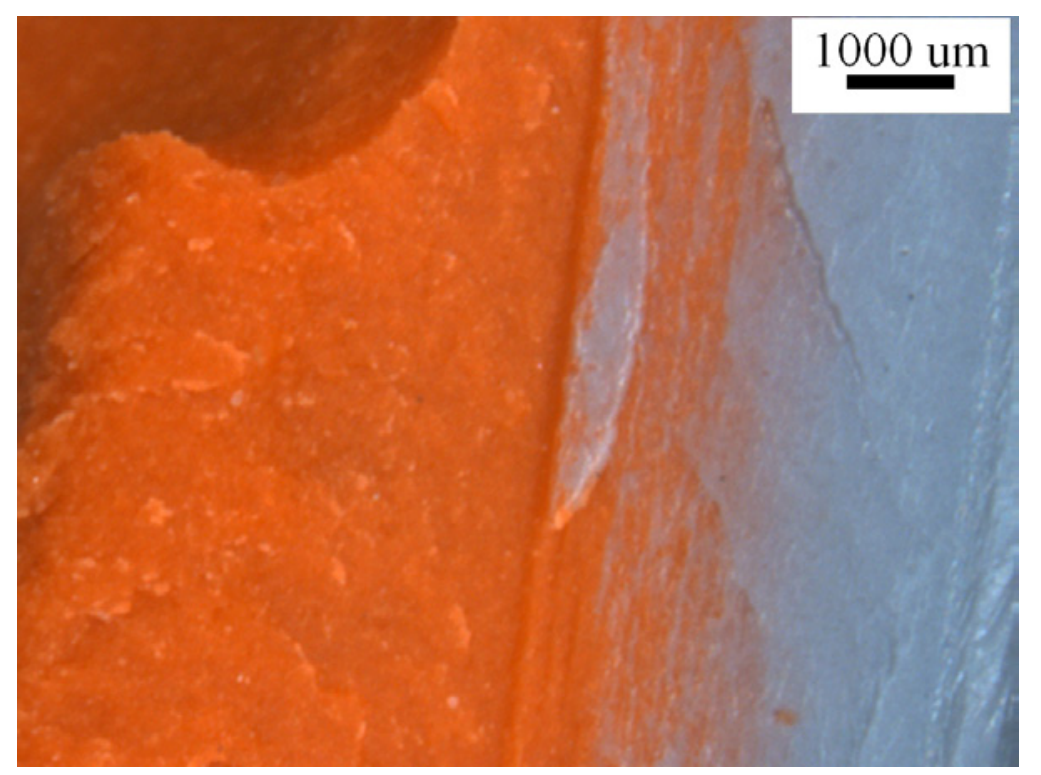

Figure 4.21: Optical micrograph of S0383-70 material failure (M) under 20X magnification near a geometric stress concentration

Figure 4.22 shows the progression of failure along the seal bulb. It is visible as a series of tear lines arcing from the edge of the crown. This type of surface is noticeable on sections of the surface created by the test shown in Figure 4.23, in which the elastomer peeled off the top of the crown at a slight angle along a span of several inches. 


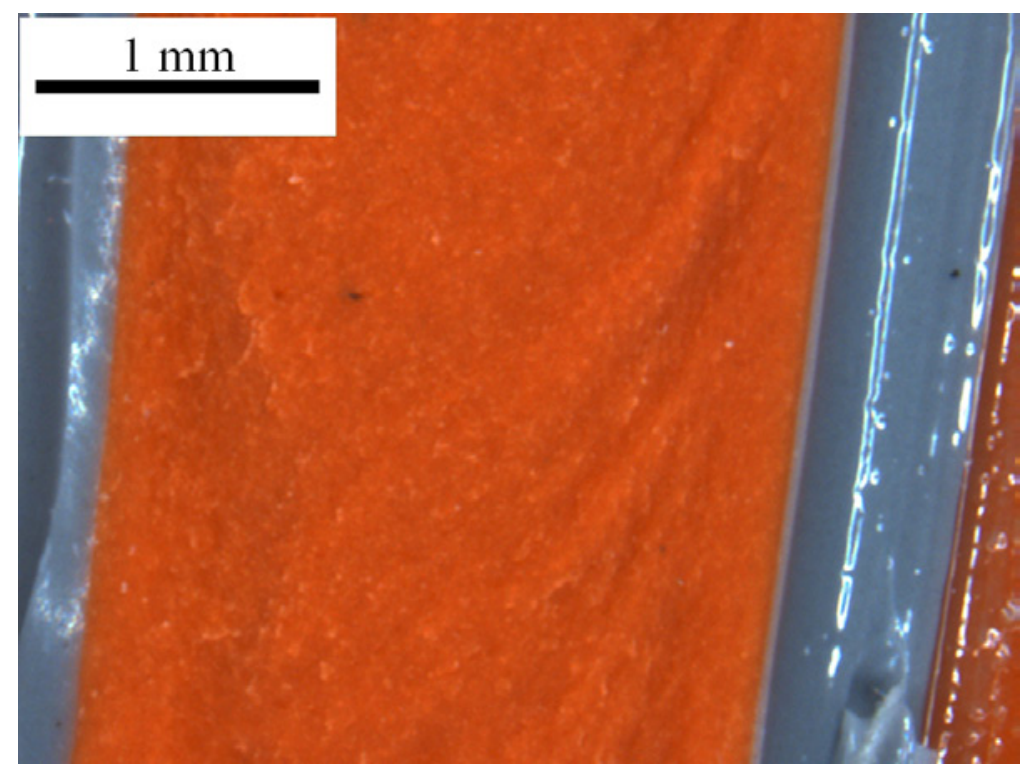

Figure 4.22: Optical micrograph of material fracture (M) surface under 16X magnification showing tear lines along seal bulb

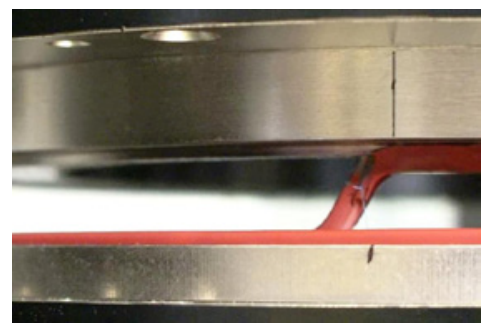

(a) Frame 1

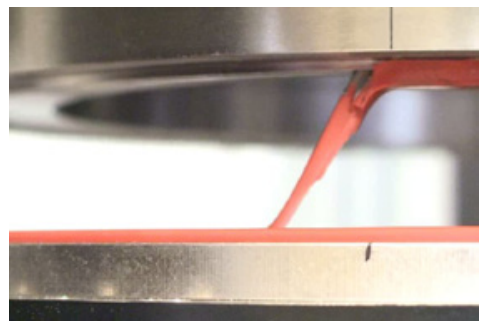

(b) Frame 2

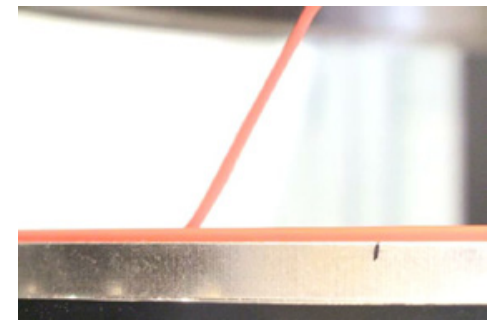

(c) Frame 3

Figure 4.23: Video frame sequence showing the progressive material failure along the seal crown

\section{Data Analysis}

The results from three localized adhesion case tests which demonstrate failure in the seal material are plotted in Figure 4.24. Similar to the uniform adhesion case data, all three curves correspond closely in the initial approximately linear region. The "sawtooth" pattern evident in both the inner and outer unflawed seal tests is characterized by start-stop failure. Failure progresses for a distance, then stalls until enough stress builds up again to recommence the propagation of the separation. 


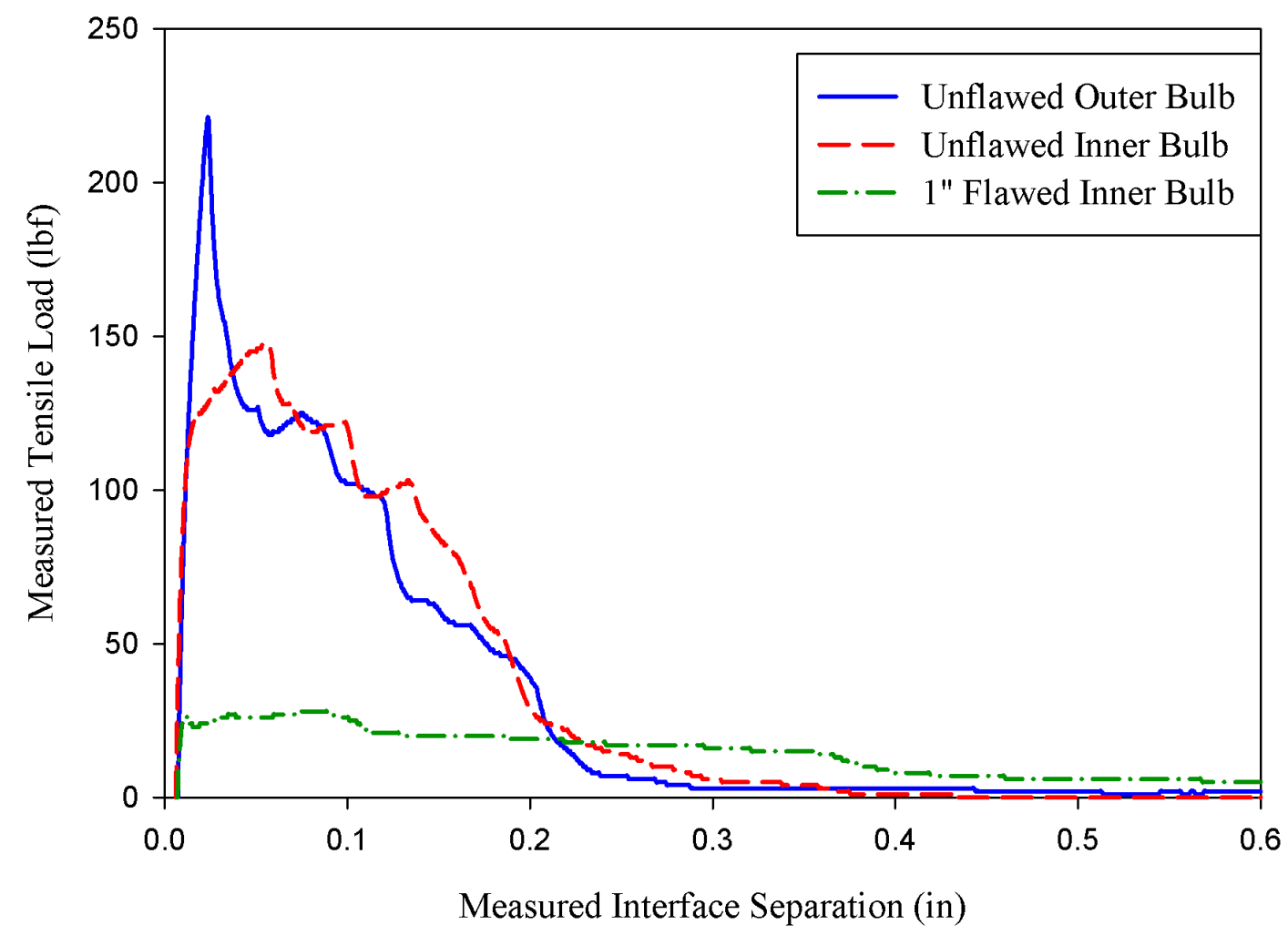

Figure 4.24: Graph of measured results for localized adhesion case exhibiting S0383-70 material failure

The behavior of the curve for the 1 in. flawed specimen relates to the unpredictability of failure propagation in elastomer materials discussed in Section 4.4.2. Instead of the failure beginning in the bond region and commencing along the circumference as was the case in the adhesive failure mode, the failure propagated upwards into the seal bulb. Upon reaching the base of the crown, the failure continued on a slight angle upwards until finally it reached the surface of the crown and separated completely. The surface of this unexpected change in direction for the failure is shown in Figure 4.25. Intuition suggests that the bond must have been unusually strong at the edge of the disbonded area in order for the failure on both sides of the disbond to travel through the material instead. This result is in stark contrast to the low load 
required to peel out the seal on the other 1 in. flawed seal bulb tested.

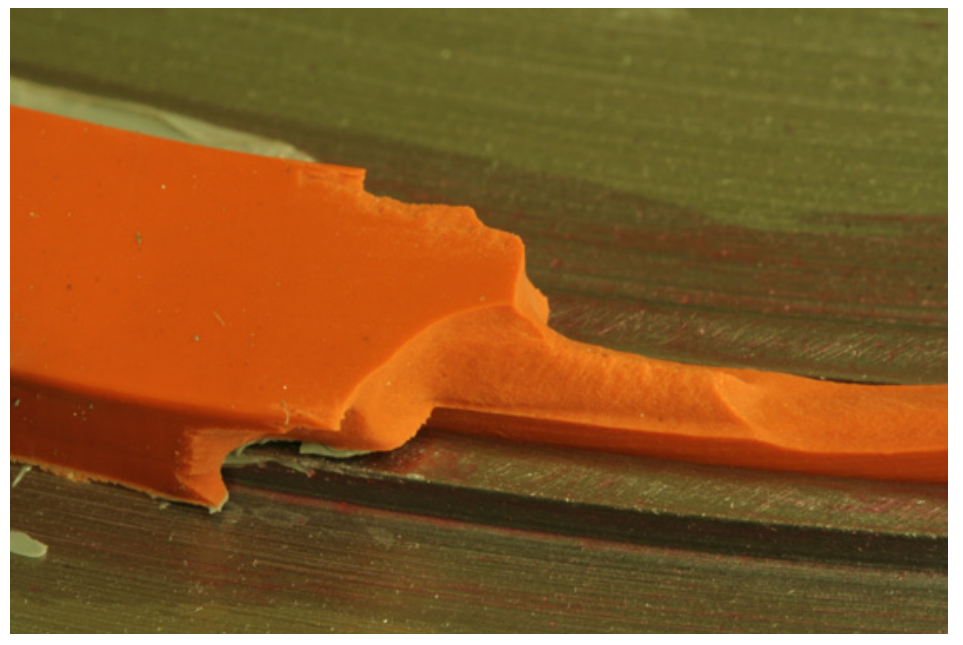

Figure 4.25: Photograph of failure surface of 1 in. disbonded seal specimen with unexpected failure propagation

\section{References}

[1] American Society for Testing and Materials, West Conshohocken, PA. Standard Test Methods for Rubber PropertyAdhesion to Rigid Substrates, 2008.

[2] American Society for Testing and Materials, West Conshohocken, PA. Standard Test Method for Adhesion-in-Peel of Elastomeric Joint Sealants, 2006.

[3] E. M. Petrie. Handbook of Adhesives and Sealants, pages 7-8. McGraw-Hill Professional, 2nd edition, 2006. 


\section{Chapter 5}

\section{Conclusions}

Composite seals have not been proven in a docking role in the history of manned space flight. One of the key concerns with the composite seal concept is the reliance on a molded bond between the elastomer and metal retainer for seal retention during interface separation rather than physically restraining features. As a result, bond strength between the elastomer and metal is an important performance characteristic that must be considered for a successful seal design. The combination of measured load capacity of the bond and the observed manner of failure is key to the description of silicone-to-metal bond strength. This work reveals that the current leading candidate design for the LIDS main interface seal under the nominal fabrication process (unflawed) exhibits adequate load capacity but an undesirable mode of failure. In seals with intentional flaws, the flaw sizes do not have a strong influence on the bond strength.

\subsection{Summary of Results}

The bond strength experimental results are presented both quantitatively through analysis of collected data and through qualitative observation of the fracture surfaces. The experimental results for the uniform adhesion case reveal a range of cohesive, adhesive, and material failure modes. The bond ratings of specimens in this case 
range from $99 \%$ adhesion, $1 \%$ cohesion to $80 \%$ adhesion, $20 \%$ cohesion, leading to the conclusion that the bond formed in the current fabrication process does not produce complete cohesive failure, which is the ideal bond failure mode. Optical microscopy of a cohesive/adhesive mix failure surface reveals that there can be smaller regions of cohesive failure not visible to the naked eye. The same is not found with the adhesively failed seal specimens. Comparison with representative seal-on-plate adhesion tests of other subscale seals reveals two orders of magnitude of difference between the adhesive load the elastomer material can be expected to produce and the necessary load to damage or dislodge the seal bulb. However, future tests may reveal conditions that substantially increase the adhesion generated by the S0383-70 silicone, and slimmer margins may be identified. The implication of these comparisons is that an acceptable margin must be decided as part of the acceptance of the final seal design.

The localized adhesion case is not directly comparable to other LIDS seal testing and thus the nature of failure is the key component for judgment of bond strength. The results of the localized adhesion tests show adhesive peel-out of the entire bulb as well as material failure. Material failure is the more desirable of these failure modes because it would not guarantee the loss of both sealing ability and redundancy in the LIDS system as the complete pull-out scenario would. The load profile applied in this case is a true worst-case scenario because it concentrates the load heavily on a thin peel line. It leads to the conclusion that once peel has begun, very low load is needed to remove the remaining elastomer from the groove.

In the uniform adhesion case, a seal bulb which failed adhesively showed a similar load-displacement pattern as the cohesively failed tests, as well as a high peak load. This result leads to the observation that bonds that fail adhesively are not necessarily too weak for the application requirements.

Neither load case tested revealed a strong correlation between the size of disbond and the failure load measured, nor with the failure mode witnessed. For the uniform adhesion case, it is proposed that the small disbond size relative to the circumference of the seal leads to failure occuring elsewhere and propagating though the bonded 
area before enough deflection can occur to stretch the disbonded section of the bulb and concentrate load at the peel line. In the localized adhesion test case, the lack of a relationship between load measured and flaw level suggest that material and bond variability between such small areas of different specimens is more influential than the length of disbond.

\subsection{Future Work}

In the development of the LIDS Gask-O-Seal ${ }^{T M}$ design, the different failure modes and their corresponding load capacities will play a role in the acceptance of a final design. Once data is produced from testing under the highest expected adhesion conditions (e.g. long-term mating), an acceptable margin can be defined between the bond failure loads reported in this work and adhesive loads generated by the compression of the seal. Adjustments to the design may be needed to increase the bond rating toward more cohesive failure including changes to the way the grooves are plated or treated during fabrication. The silicone-to-metal bond strength of the composite main interface seal is a crucial consideration in the design of the LIDS. 


\section{Appendix A}

\section{Measurement Uncertainty}

Measured load-displacement data represents the quantitative element in the multifaceted approach in understanding bond strength of the LIDS main interface seal. Force is measured using a load cell integrated with the load frame, and displacement is measured using both an integrated position encoder and an external laser extensometer. These devices have uncertainties associated with the measurements they produce which require some consideration.

The displacement measurements are subject to uncertainties arising from the physical test setup and from the instrumentation itself. The most notable physical source of uncertainty is during the plate shifting phenomenon observed on several of the particularly high load cases. In these tests, the interface is not necessarily at the displacement recorded by the position encoder because there is angular deflection occurring. Also, the laser extensometer is only measuring the interface at one location and thus can not distinguish the direction of deflection, nor does it perceive whether it is at the high end or low end. However, the displacement measurement is not of prime interest as it relates to bond strength in this research.

The uncertainty associated with the load measurement is of real interest. Prior to testing, the load cell was calibrated to a maximum error within $0.29 \%$ of the reading (in tension). Despite this high accuracy, the nature of the subscale destructive 
tests introduces a much larger source of uncertainty. The bond strength load that is measured by the load cell during separation is not necessarily acting uniformly around the seal circumference. In fact, it is most likely acting at some distance off-axis from the center of the seal. This results in a bending moment on the load frame rod, which then transfers into the load cell as shear load, distorting the measurement. The effect on the measurement of this off-center load is given by the load cell manufacturer as $0.5 \%$ per $1 \mathrm{~cm}$ off-axis. For an inner and outer bulb, this equates to $6.3 \%$ and $7.5 \%$, respectively, as the maximum amount of error that could be introduced by off-axis loading.

The critical point here is that the resultant force that the load cell measures is not actually acting at the extreme outer radius, but these uncertainty estimates bound the error that could be present due to off-axis loading. The maximum uncertainty estimates are rounded and used for both direct load measurements and calculated load per inch values for comparisons (see Figure 4.8). The uncertainty in the circumference used in the calculation is negligible, as shown in the following calculations:

The load per circumferential inch is calculated as $F_{\text {linear }}=\frac{f_{\text {peak }}}{\pi D_{\text {bulb }}}$, where $f_{\text {peak }}$ is the measured load at failure initiation and $D_{b u l b}$ is the diameter of the bulb being tested. To calculate the combined error from the load measurement and the seal diameter, the following error propagation formula can be used. ${ }^{1}$

$$
\frac{\delta F_{\text {linear }}}{F_{\text {linear }}}=\sqrt{\frac{\delta f_{\text {peak }}}{f_{\text {peak }}}+\frac{\delta D_{\text {bulb }}}{D_{\text {bulb }}}}
$$

The ratio of error in the diameter to its value, $\frac{\delta D_{b u l b}}{D_{b u l b}}$, is $0.05 \%$. Therefore, the same values for uncertainty stemming from the off-axis load are used for the load per inch values.

Although the maximum possible error is $7 \%$, much higher variability can be expected simply due to the stochastic nature of fracture. Material variations, different bond conditions, and a host of other factors can significantly affect the measurements taken, so that the maximum possible error is still well within the variability expected 
due to uncontrolled parameters.

\section{References}

[1] J. R. Taylor. An Introduction to Error Analysis. University Science Books, 2nd edition, 1982. 


\section{Bibliography}

NASA's Exploration Systems Architecture Study Final Report, 2005.

American Society for Testing and Materials, West Conshohocken, PA, Standard test method for adhesion-in-peel of elastomeric joint sealants, 2006.

American Society for Testing and Materials, West Conshohocken, PA, Standard guide for preparation of metal surfaces for adhesive bonding, 2008.

American Society for Testing and Materials, West Conshohocken, PA, Standard test methods for rubber propertyadhesion to rigid substrates, 2008.

C. C. Daniels, H. C. deGroh, III, P. H. Dunlap, Jr, J. R. Finkbeiner, B. M. Steinetz, M. B. Bastrzyk, J. J. Oswald, B. A. Banks, J. A. Dever, S. K. Miller, and D. L. Waters, Characteristics of elastomer seals exposed to space environments, 43rd AIAA/ASME/SAE/ASEE Joint Propulsion Conference and Exhibit, no. AIAA 2007-5741, 2007.

C. C. Daniels, J. J. Oswald, M. B. Bastrzyk, I. Smith, P. H. Dunlap, Jr, and B. M. Steinetz, Experimental investigation of elastomer docking seal compression set, adhesion, and leakage, AIAA SPACE 2007 Conference and Exposition, no. AIAA 2007-6197, 2007.

C. C. Daniels, J. J. Oswald, P. H. Dunlap, Jr, and B. M. Steinetz, Elastomeric seal development of Advanced Docking/Berthing System, 2005 NASA Seal/Secondary Air System Workshop, 2005, pp. 465-484. 
H. C. deGroh, III, C. C. Daniels, P. H. Dunlap, Jr, S. Miller, J. Dever, D. Waters, J. J. Oswald, and B. M. Steinetz, Space environment's effects on seal materials, 2006 NASA Seal/Secondary Air System Workshop, 2006, pp. 507-523.

H. C. deGroh, III, S. K. R. Miller, I. Smith, C. C. Daniels, and B. M. Steinetz, Adhesion of silicone elastomer seals for NASA's Crew Exploration Vehicle, Tech. Report NASA TM-2008-215433, National Aeronautics and Space Administration, 2008.

J. R. Finkbeiner, P. H. Dunlap, Jr, B. M. Steinetz, and C. C. Daniels, Review of seal designs on the Apollo spacecraft, Journal of Spacecraft and Rockets 45 (2008), no. 5 .

Erik Illi, Space station freedom common berthing mechanism, Proceedings of the 26th Aerospace Mechanisms Symposium, 1992, pp. 281-296.

J. L. Lewis, M. B. Carroll, R. H. Morales, and D. L. Thang, Androgynous, reconfigurable closed loop feedback controlled Low Impact Docking System with load sensing electromagnet capture system, 2002.

J. J. Oswald, C. C. Daniels, P. H. Dunlap, Jr, and B. M. Steinetz, Metallic seal development of Advanced Docking/Berthing System, 2005 NASA Seal/Secondary Air System Workshop, 2005, pp. 507-523.

E. M. Petrie, Handbook of Adhesives and Sealants, 2nd ed., pp. 7-8, McGraw-Hill Professional, 2006.

I. Smith and C. C. Daniels, Experimental investigation into medium scale LIDS seal performance, 2007 NASA Seal/Secondary Air System Workshop, 2007.

W. L. Swan, Jr, Apollo-Soyuz Test Project docking system, Proceedings of the 10th Aerospace Mechanisms Symposium, 1976, pp. 26-37. 
J. R. Taylor, An Introduction to Error Analysis, 2nd ed., University Science Books, 1982. 